\title{
Three-Manifolds Having Complexity At Most 9
}

\author{
Bruno Martelli and Carlo Petronio
}

\section{CONTENTS}

1. Introduction

2. The Complexity Function

3. The Algorithm to Find Bricks

4. Minimal Skeleta of Bricks

5. Bricks and Skeleta up to Complexity 9

Electronic Availability

References
We describe an algorithm which has enabled us to give a complete list, without repetitions, of all closed oriented irreducible three-manifolds of complexity up to 9. More interestingly, we have actually been able to give a name to each such manifold, by recognizing its canonical decomposition into Seifert fibered spaces and hyperbolic manifolds.

The algorithm relies on the extension of Matveev's theory of complexity to the case of manifolds bounded by suitably marked tori, and on the notion of assembling of two such manifolds. We show that every manifold is an assembling of manifolds which cannot be further disassembled, and we prove that there are surprisingly few such manifolds up to complexity 9.

Our most interesting experimental discovery is that there are 4 closed hyperbolic manifolds having complexity 9, and they are the 4 closed hyperbolic manifolds of least known volume. All other manifolds having complexity at most 9 are graph manifolds.

\section{INTRODUCTION}

This paper is devoted to the theoretical description and illustration of results of an algorithm which has enabled us to give a complete list, without repetitions, of all closed oriented irreducible 3-manifolds of complexity up to 9 . More interestingly, we have actually been able to give a "name" to each such manifold, that is, to recognize its canonical decomposition into Seifert fibered spaces and hyperbolic manifolds already considered by other authors. The complexity we have in mind is that introduced by Matveev [1990], given by the minimal number of vertices of a simple spine (and, as Matveev proved in the same work, equal to the minimal number of tetrahedra in a triangulation). See also [Matveev and Fomenko 1988].

Our algorithm relies on a structural result on closed three-manifolds. Namely, we show that all closed three-manifolds can be obtained by combining, in a suitable sense, building blocks taken from a certain 
list which, at least up to complexity 9 , is dramatically shorter than the list of all manifolds. The building blocks are called bricks, they are bounded by tori, and these tori carry a "marking" given by an embedded trivalent graph. Moreover, the combination of two bricks corresponds to the identification of two boundary tori. The main definitions and results of the theory of decomposition into bricks are stated in the rest of the present introduction and proved in the body of the paper.

Before turning to bricks, we mention the most interesting experimental results about complexity 9 which our algorithm has allowed us to discover. Recall first that it was already known to Matveev [1990] that up to complexity 8 all manifolds are graph-manifolds; tables up to complexity 6 are in [Matveev 1998], and up to 7 in [Ovchinnikov 1997]. Now, we can show that there are 1156 manifolds of complexity nine, 272 of them are lens spaces, 863 are more general graph-manifolds which do not contain nonseparating tori, 17 of them are torus bundles over $S^{1}, 10$ of them are graph-manifolds with graph $\mathrm{O} \cdot$, and there are also 4 closed hyperbolic manifolds. More importantly, these 4 manifolds turn out to be precisely those of least known volume [Hodgson and Weeks 1994], in accordance with the ideas about complexity and volume stated in [Matveev and Fomenko 1988].

\section{A. Bricks and Assemblings of Bricks}

We will work throughout in the PL category, and by manifold we will always mean a compact orientable three-manifold, possibly with boundary. We will call triod the graph with two vertices and three edges all joining one vertex to the other one. Note that a triod $\theta$ can be embedded in a torus $T$ so that $T \backslash \theta$ is an open 2-disc. A pair $(M, X)$ is said to be a manifold with triods if $M$ is a manifold with boundary consisting of tori $T_{1}, \ldots, T_{n}$ and $X$ is a set of triods $\left\{\theta_{1}, \ldots, \theta_{n}\right\}$, with $\theta_{i}$ embedded in $T_{i}$ so that $T_{i} \backslash \theta_{i}$ is a disc. The case where $n=0$ and $X=\varnothing$, so $M$ is closed, is admitted.

Let $X$ be the set of all manifolds with triods (up to equivalence induced by homeomorphism of manifolds). If $M$ has nonempty boundary consisting of tori, then there are infinitely many inequivalent ways to embed triods in these tori, so there are infinitely many inequivalent pairs $(M, X)$ based on the same $M$. On the contrary, if $M$ is closed, then there is a unique element $(M, \varnothing) \in \mathcal{X}$ based on $M$. Therefore the set of all closed orientable manifolds can be viewed as a subset of $X$.

We will now describe three operations on $X$ and state the crucial properties of a complexity function on $X$ introduced and discussed in detail below in Section 2.

Connected sum. The operation of connected sum "far from the boundary" obviously extends from manifolds to manifolds with triods. Namely, given $(M, X)$ and $\left(M^{\prime}, X^{\prime}\right)$ in $X$, we define $(M, X) \#\left(M^{\prime}, X^{\prime}\right)$ as $\left(M \# M^{\prime}, X \cup X^{\prime}\right)$, where $M \# M^{\prime}$ is one of the two possible connected sums of $M$ and $M^{\prime}$ (recall that our manifolds are orientable but not oriented). Of course $\left(S^{3}, \varnothing\right) \in \mathcal{X}$ is the identity element for operation \#. We will call a pair $(M, X)$ prime if $M$ is; that is, if $(M, X)$ cannot be expressed as a connected sum of pairs different from $\left(S^{3}, \varnothing\right)$.

Assembling. Given $(M, X)$ and $\left(M^{\prime}, X^{\prime}\right)$ in $X$, we pick triods $\theta_{i} \in X$ and $\theta_{i^{\prime}}^{\prime} \in X^{\prime}$ and choose a homeomorphism $\psi: T_{i} \rightarrow T_{i^{\prime}}^{\prime}$ such that $\psi\left(\theta_{i}\right)=\theta_{i^{\prime}}^{\prime}$. We can then construct the manifold with triods $(N, Y)=$ $\left(M \cup_{\psi} M^{\prime},\left(X \cup X^{\prime}\right) \backslash\left\{\theta_{i}, \theta_{i^{\prime}}^{\prime}\right\}\right)$. We call this an assembling of $(M, X)$ and $\left(M^{\prime}, X^{\prime}\right)$ and we write $(N, Y)=(M, X) \oplus\left(M^{\prime}, X^{\prime}\right)$. Of course two given elements of $X$ can only be assembled in a finite number of inequivalent ways.

Operation $\oplus$ has an identity element, and in a special case it is the inverse operation of \#. Below we will need to exclude these types of assembling, so we describe them in detail. First, set

$$
B_{0}=(T \times[0,1],\{\theta \times\{0\}, \theta \times\{1\}\}),
$$

where $T$ is the torus and $\theta \subset T$ is a triod such that $T \backslash \theta$ is a disc ( $B_{0}$ is well-defined up to equivalence). Of course if we assemble any $(M, X) \in X$ with $B_{0}$ we get $(M, X)$ again.

Let $H$ be the solid torus and let $(H,\{\theta\})$ and $\left(H,\left\{\theta^{\prime}\right\}\right)$ be elements of $X$ based on $H$. Assume that there exists a homeomorphism $\partial H \rightarrow \partial H$ with such that $\psi(\theta)=\theta^{\prime}$ and that performing the assembling $(H,\{\theta\}) \oplus\left(H,\left\{\theta^{\prime}\right\}\right)$ along $\psi$ gives $\left(S^{3}, \varnothing\right)$ as a result. Then for any $(M, X) \in \mathcal{X}$ we have

$$
((M, X) \#(H,\{\theta\})) \oplus\left(H,\left\{\theta^{\prime}\right\}\right)=(M, X)
$$

if we use the same $\psi$. 
This discussion motivates the following definition. An assembling $(M, X) \oplus\left(M^{\prime}, X^{\prime}\right)$ is called trivial if, up to interchanging $(M, X)$ and $\left(M^{\prime}, X^{\prime}\right)$, one of the following holds:

- $\left(M^{\prime}, X^{\prime}\right)=B_{0}$, or

- $\left(M^{\prime}, X^{\prime}\right)=\left(H,\left\{\theta^{\prime}\right\}\right)$ is a solid torus with triod, and $(M, X)$ can be decomposed as $(M, X)=$ $(N, Y) \#(H,\{\theta\})$ so that $(N, Y) \neq\left(S^{3}, \varnothing\right)$ and the assembling identifies $\theta$ to $\theta^{\prime}$ and $(H,\{\theta\}) \oplus$ $\left(H,\left\{\theta^{\prime}\right\}\right)=\left(S^{3}, \varnothing\right)$.

Self-assembling. Given $(M, X) \in \mathcal{X}$, we pick two distinct triods $\theta_{i}, \theta_{i^{\prime}}$ in $X$, we choose a homeomorphism $\psi: T_{i} \rightarrow T_{i^{\prime}}$ such that $\psi\left(\theta_{i}\right)$ and $\theta_{i^{\prime}}$ intersect transversely in two points, and we construct the manifold with triods

$$
(N, Y)=\left(M_{\psi}, X \backslash\left\{\theta_{i}, \theta_{i^{\prime}}\right\}\right) .
$$

We call this a self-assembling of $(M, X)$ and we write $(N, Y)=\odot(M, X)$. As above, only a finite number of self-assemblings of a given element of $X$ are possible.

In the sequel it will be convenient to refer to a combination of assemblings and self-assemblings of pairs just as an assembling. Note that of course we can do the assemblings first and the self-assemblings in the end.

A complexity on $X$. One of the main ingredients of the present paper is the extension of Matveev's definition of complexity [1990] from closed manifolds to manifolds with triods. We warn the reader that Matveev's complexity $c(M)$ is defined also when $M$ has nonempty boundary, but our definition will be different in this case, namely we will have $c(M, X)=$ $c(M)$ only when $X=\varnothing$; that is, when $M$ is closed. The key properties of $c$, proved below, are additivity with respect to connected sum and subadditivity with respect to assembling. More precisely, we will construct in Section 2A a function $c: X \rightarrow \mathbb{N}$ and show in Section 2B that it enjoys the following properties:

1. $c(M, \varnothing)=c(M)$ for any $(M, \varnothing) \in X$;

2. $c\left((M, X) \#\left(M^{\prime}, X^{\prime}\right)\right)=c(M, X)+c\left(M^{\prime}, X^{\prime}\right)$;

3. $c\left((M, X) \oplus\left(M^{\prime}, X^{\prime}\right)\right) \leq c(M, X)+c\left(M^{\prime}, X^{\prime}\right)$, and if equality holds and the assembling is nontrivial, then $(M, X) \oplus\left(M^{\prime}, X^{\prime}\right)$ is prime if and only if both $(M, X)$ and $\left(M^{\prime}, X^{\prime}\right)$ are;
4. $c(\odot(M, X)) \leq c(M, X)+6$, and if equality holds, $\odot(M, X)$ is prime if and only if $(M, X)$ is;

5. for any $n \geq 0$ there is only a finite number of prime pairs $(M, X) \in X$ with $c(M, X) \leq n$.

Now let $X^{\text {pr }} \subset X$ be the set consisting of prime pairs. An assembling is called sharp if it is nontrivial and the inequality of point (3) above is actually an equality. Similarly, a self-assembling is sharp if in (4) we have an equality. We will say that a prime pair $(M, X) \in X^{\mathrm{pr}}$ is a brick if it cannot be expressed as the result of a sharp assembling or a sharp selfassembling. The following easy result will be proved in Section 2A (one could actually also deduce it from property (5), but we will refrain from doing this):

Lemma 1.1. The pair $B_{0}$ is the only $(M, X) \in X$ such that $c(M, X)=0$ and $X$ contains at least two triods.

Induction on complexity now readily implies the following:

Proposition 1.2. Every prime manifold with triods can be obtained as a sharp-assembling of some bricks.

We define now $\mathcal{B} \subset X^{\mathrm{pr}}$ as the set of all bricks, and note that $\mathcal{B}$ naturally splits as $\mathcal{B}^{0} \sqcup \mathcal{B}^{1}$, where $\mathcal{B}^{0}$ is the set of all $(M, X) \in \mathcal{B}$ with $X=\varnothing$ (that is, $M$ is closed). Pairs in $\mathcal{B}^{0}$ cannot be used for an assembling or self-assembling, since they have no boundary. Let $\mathcal{B}_{n}^{j} \subset \mathcal{B}^{j}$, for $j=0,1$, and $X_{n} \subset X$ be the subsets consisting of pairs having complexity $n$. Proposition 1.2 and the properties of $c$ stated above now imply that

$$
\begin{aligned}
X_{\leq n}^{\mathrm{pr}}=\mathcal{B}_{\leq n}^{0} \cup\left\{\bigodot^{k}\left(B^{1} \oplus \cdots \oplus B^{h}\right):\right. \\
\left.B^{i} \in \mathcal{B}_{\leq n}^{1}, \sum c\left(B^{i}\right)+6 k \leq n\right\} .
\end{aligned}
$$

If one can give an unambiguous name to each closed $\odot^{k}\left(B^{1} \oplus \cdots \oplus B^{h}\right)$, then the set of all closed prime manifolds having complexity at most $n$ is easily constructed from $\mathcal{B}_{\leq n}$ by listing the (finite number of) closed manifolds obtained in this way, and by then removing duplicates. For $n \leq 9$ it turns out that $\mathcal{B}_{\leq n}$ consists of a very few atoroidal manifolds (with triods), and it is experimentally not so hard to give a name to each closed manifold of the form $\odot^{k}\left(B^{1} \oplus \cdots \oplus B^{h}\right)$. We will provide more details below on the recognition issue (after listing the bricks explicitly), but we want to emphasize here that the 


\begin{tabular}{|ccccc|}
\hline$(M, X)$ & $c(M, X)$ & $M$ & $X$ & $c(M)$ \\
\hline$B_{0}$ & 0 & $D_{1} \times S^{1}$ & $\{(1,0),(0,1),(-1,-1)\}$ & 0 \\
& & & $\{(1,0),(0,-1),(-1,1)\}$ & \\
$B_{1}$ & 0 & $D_{0} \times S^{1}$ & $\{(1,0),(0,1),(-1,-1)\}$ & 0 \\
$B_{2}$ & 0 & $D_{0} \times S^{1}$ & $\{(0,1),(1,1),(-1,-2)\}$ & 0 \\
$B_{3}$ & 1 & $D_{1} \times S^{1}$ & $\{(1,0),(0,1),(-1,-1)\}$ & 0 \\
& & & $\{(1,0),(0,1),(-1,-1)\}$ & \\
$B_{4}$ & 3 & $D_{2} \times S^{1}$ & $\{(1,0),(0,1),(-1,-1)\}$ & 0 \\
& & & $\{(1,0),(0,1),(-1,-1)\}$ & \\
$B_{5}$ & 8 & $\left(D_{0},(2,1),(3,1)\right)$ & $\{(1,0),(0,-1),(-1,1)\}$ & \\
$B_{6}$ & 8 & $M 2_{2}^{1}$ & $\{(1,0),(0,-1),(-1,1)\}$ & 2 \\
$B_{7}$ & 9 & $M 3_{4}^{1}$ & $\{(1,0),(0,-1),(-1,1)\}$ & 3 \\
$B_{8}$ & 9 & $M 4_{1}^{2}$ & $\{(1,0),(0,1),(-1,-1)\}$ & 4 \\
& & & $\{(1,0),(0,1),(-1,-1)\}$ & \\
$B_{9}$ & 9 & $M 6_{1}^{3}$ & $\{(1,0),(0,-1),(-1,1)\}$ & 6 \\
& & & $\{(1,0),(0,-1),(-1,1)\}$ & \\
$B_{10}$ & & & $\{(1,0),(0,-1),(-1,1)\}$ & \\
& 9 & & $\{(1,0),(0,-1),(-1,1)\}$ & 6 \\
& & & $\{(1,0),(0,-1),(-1,1)\}$ & \\
& & & $\{(1,0),(0,1),(-1,-1)\}$ & \\
& & &
\end{tabular}

TABLE 1. Bricks up to complexity 9.

vast majority of computer time in the implementation of our algorithm was taken by the determination of bricks. Taking the list of bricks for granted, the reader could with some patience reproduce the list of manifolds by himself.

\section{B. Bricks and Manifolds up to Complexity 9}

The algorithm that will be explained in Section 3 has enabled us to explicitly find $\mathcal{B}_{\leq 9}^{0}$ and $\mathcal{B}_{\leq 9}^{1}$. The former consists of 19 closed manifolds that naturally come in two families $C_{i, j}$ and $E_{k}$, and the latter consists of only 11 manifolds with triods, denoted by $B_{0}, \ldots, B_{10}$ (where $B_{0}$ is the same as defined above). The elements of $\mathcal{B}_{<9}^{0}$ are all Seifert fibered over $S^{2}$ with 3 exceptional fibers. In order to describe the elements of $\mathcal{B}_{\leq 9}^{1}$ we need a way to encode the possible ways a triod can sit in a torus.

Remark 1.3. Let $T$ be a torus. Let $\mathcal{T}$ be the set of unordered triples $\{a, b, c\}$ of elements of $H_{1}(T)$, such that every pair of elements in $\{a, b, c\}$ generates $H_{1}(T)$, and $a+b+c=0$. Let $\theta \subset T$ be a triod such that $T \backslash \theta$ is a disc: inside $\theta$ we can find 3 distinct closed curves, which can be oriented in order to form a triple $\{a, b, c\} \in \mathcal{T}$. The only two triples we can get like this are $\{a, b, c\}$ and $\{-a,-b,-c\}$. Conversely, each triple $\{a, b, c\} \in \mathcal{T}$ determines a triod $\theta \subset T$. It follows that triods (up to isotopy) are in one-toone correspondence with elements of $\mathcal{T} / \mathbb{Z}_{2}$, where the nontrivial element of $\mathbb{Z}_{2}$ acts mapping $\{a, b, c\}$ to $\{-a,-b,-c\}$.

Bricks. In Table 1 we list the elements in $\mathcal{B}_{\leq 9}^{1}$, as produced by our algorithm, where $D_{k}$ is the disc with $k$ holes, and the usual notation for Seifert manifolds and cusped hyperbolic manifolds [Callahan et al. 1999] is employed. Note that $c(M) \neq c(M, X)$ is the complexity of $M$ in the usual sense [Matveev 1990], defined for any compact three-manifold. Every $M$ turns out to be atoroidal. In order to describe triods as elements in $\mathcal{T}$, we must fix a basis $\left(\mu_{i}, \lambda_{i}\right)$ for $H_{1}\left(T_{i}\right)$ for each $T_{i}$ in $\partial M$. When $M$ is Seifert, by removing fibered neighbourhoods of the exceptional fibers we get $D_{k} \times S^{1}$ with the product fibration. Then we choose $\lambda_{i}$ to be a fiber and $\mu_{i}$ to be a component of $\partial D_{k} \times\{$ point $\}$, with orientations chosen 
so that $\left(\mu_{i}, \lambda_{i}\right)$ is a positively oriented basis. When $M$ is hyperbolic we choose $\mu_{i}$ and $\lambda_{i}$ to be the basis used by SnapPea. In both cases, taking $\left(-\mu_{i},-\lambda_{i}\right)$ instead of $\left(\mu_{i}, \lambda_{i}\right)$ as a basis does not make any difference, since triples in $\mathcal{T}$ are defined up to sign.

From bricks to manifolds. As pointed out above, a list of all closed orientable prime manifolds with complexity at most 9 can be compiled by listing and recognizing all closed manifolds obtained by assembling bricks $B^{1}, \ldots, B^{h}$ of $\mathcal{B}_{\leq 9}$ and then self-assembling $k$ times, with $\sum c\left(B^{i}\right)+6 k \leq 9$. We explain here the points which make this listing and recognition feasible. Note first that, by the bound on complexity, only a few assemblings, and no self-assembling, will involve $B_{5}, \ldots, B_{10}$. We also know that $B_{0}$ must not be used for assemblings. Moreover we can eliminate from the list all assemblings which we know a priori not to be sharp. For instance we have the following (proved in Section 2):

Proposition 1.4. If $(M, X) \in X^{\mathrm{pr}}$ and $(M, X) \oplus B_{1}$ is sharp, then $(M, X)$ is either $B_{1}$ or $B_{2}$.

Concerning recognition, we note now that the effect of assembling $B_{2}$ or $B_{3}$ is very easy to describe. Since $B_{2}$ is a solid torus, the assembling with $B_{2}$ along some boundary component $T_{i}$ corresponds to a Dehn filling of $T_{i}$. Finitely many different fillings are possible, and they are determined by the position in $T_{i}$ of the triod $\theta_{i}$. Now

$$
B_{3} \cong\left(T \times[0,1],\left\{\theta_{0} \times\{0\}, \theta_{1} \times\{1\}\right\}\right)
$$

with $\theta_{0} \neq \theta_{1}$. (Even if in Table 1 the triples describing the triods are the same, the triods are not the same, because they lie on different boundary components, so the bases of homology are different due to orientation.) More precisely, the assembling with $B_{3}$ along $T_{i}$ corresponds to changing the position of the triod $\theta_{i}$ as in Figure 1. Summing up, the successive assembling along $T_{i}$ of some $B_{3}$ 's followed by the assembling of one $B_{2}$ still corresponds to a Dehn filling of $T_{i}$. One actually sees that all Dehn fillings can be generated like this, but of course the bound on complexity allows to consider only finitely many of them.

Turning to $B_{4}$ and $B_{5}$, we note that they naturally come with a Seifert fibered structure, so any manifold generated by $B_{2}, \ldots, B_{5}$ is a graph manifold, whose graph and gluing matrices are easily deduced

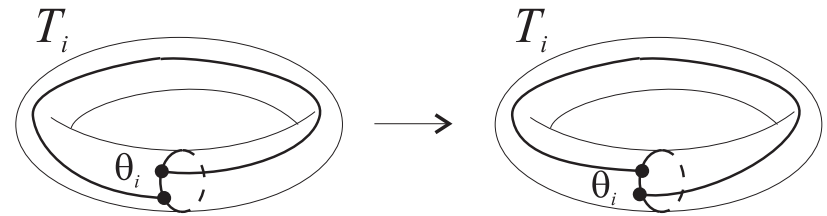

FIGURE 1. The effect of an assembling with $B_{3}$.

from the pattern of assemblings giving the manifold. Since there are algorithms checking whether two such set of data give the same manifold, recognition is not a problem at this level.

Getting to assemblings involving $B_{6}, \ldots, B_{10}$, one first notes that they can only be assembled with $B_{2}$ and $B_{3}$, and not in many ways. Next, one checks by direct comparison with the tables in [Hodgson and Weeks 1994] that 4 of the resulting manifolds are the 4 hyperbolic closed manifolds with least known volume. The following fact (proven in Section 5) concludes our investigation:

Proposition 1.5. Let $M$ be a closed manifold of complexity up to 9 obtained by assembling a brick in $\left\{B_{6}, \ldots, B_{10}\right\}$ and some $B_{2}$ 's and $B_{3}$ 's. Then either $M$ is one of the 4 hyperbolic manifolds just described, or the assembling is not sharp.

Manifolds. Table 2 contains the data our algorithm has allowed us to discover about closed orientable prime manifolds having complexity $c$ for $c \leq 9$. We have divided the manifolds into three groups, given respectively by the elements which may be obtained by sharp-assembling $B_{0}, \ldots, B_{10}$ but without self-assembling, by those which require a selfassembling, and by those of $\mathcal{B}^{0}$. The three groups have been further split to give a more precise idea of which bricks are needed to generate a manifold: in particular, the vast majority of manifolds (with 11 exceptions out of 1156 manifolds in complexity 9) are obtained assembling $\left\{B_{2}, B_{3}, B_{4}\right\}$, and only a few manifolds actually require a self-assembling. An important convention in the table is that manifolds already considered in a certain line are not considered again in subsequent lines: some manifolds can be split into bricks in distinct ways.

It follows from the topology of the bricks that the elements of $\left\langle B_{2}, B_{3}\right\rangle_{\text {nonself }}$ are all lens spaces, those of $\left\langle B_{2}, B_{3}, B_{4}\right\rangle_{\text {nonself }}$ and $\left\langle B_{2}, B_{3}, B_{5}\right\rangle_{\text {nonself }}$ are more general graph-manifolds whose graph is a tree, those of $\left\langle B_{3}\right\rangle_{\text {self }}$ are torus bundles over $S^{1}$ and those of 


\begin{tabular}{|rlrrrrrrrrr|}
\hline vertices & 0 & 1 & 2 & 3 & 4 & 5 & 6 & 7 & 8 & 9 \\
\hline$\left\langle B_{1}\right\rangle_{\text {nonself }}$ & 2 & & & & & & & & & \\
$\left\langle B_{2}\right\rangle_{\text {nonself }}$ & 2 & & & & & & & & & \\
$\left\langle B_{2}, B_{3}\right\rangle_{\text {nonself }}$ & & 2 & 3 & 6 & 10 & 20 & 36 & 72 & 136 & 272 \\
$\left\langle B_{2}, B_{3}, B_{4}\right\rangle_{\text {nonself }}$ & & & & & 2 & 8 & 33 & 97 & 292 & 856 \\
$\left\langle B_{2}, B_{3}, B_{5}\right\rangle_{\text {nonself }}$ & & & & & & & & & 1 & 3 \\
$\left\langle B_{2}, B_{3}, B_{6}\right\rangle_{\text {nonself }}$ & & & & & & & & & & 2 \\
$\left\langle B_{2}, B_{7}\right\rangle_{\text {nonself }}$ & & & & & & & & & & 1 \\
$\left\langle B_{2}, B_{8}\right\rangle_{\text {nonself }}$ & & & & & & & & & & 1 \\
\hline$\left\langle B_{0}\right\rangle_{\text {self }}$ & & & & & & & 4 & & & \\
$\left\langle B_{3}\right\rangle_{\text {self }}$ & & & & & & & & 3 & 3 & 7 \\
$\left\langle B_{2}, B_{4}\right\rangle_{\text {self }}$ & & & & & & & & & & 10 \\
\hline$C_{i, j}$ & & & 1 & 1 & 2 & 2 & 1 & 2 & 3 & 3 \\
$E_{k}$ & & & & & & 1 & 0 & 1 & 1 & 1 \\
\hline total & 4 & 2 & 4 & 7 & 14 & 31 & 74 & 175 & 436 & 1156 \\
\hline
\end{tabular}

TABLE 2. Manifolds up to complexity 9.

$\left\langle B_{2}, B_{4}\right\rangle_{\text {self }}$ are graph-manifolds with graph $\bigcirc \cdot$. As already mentioned, and explained in detail below in Section 5 , the elements of $\mathcal{B}^{0}$ (namely the $C_{i, j}$ 's and $E_{k}$ 's) are all Seifert fibered over $S^{2}$ with 3 exceptional fibers.

\section{THE COMPLEXITY FUNCTION}

We now extend Matveev's complexity [1990] to manifolds with triods, and we state and prove its properties.

\section{A. Definition of Complexity}

A compact polyhedron $P$ is called simple if the link of every point of $P$ can be embedded in the space given by a circle with three radii. The points having the whole of this space as a link are called vertices: they are isolated and therefore finite in number.

Let $(M, X)$ be a manifold with triods. A subpolyhedron $P$ of $M$ is said to be a skeleton of the pair $(M, X)$ if

- $P \cup \partial M$ is simple;

- $M \backslash(P \cup \partial M)$ is an open ball;

- $P \cap \partial M=X$.

Note that each open disc $T_{i} \backslash \theta_{i}$ is automatically adjacent to the ball $M \backslash(P \cup \partial M), P$ is simple, and the vertices of $P$ cannot lie on $\partial M$. Note also that when $\# X=1$ then $P$ is a spine of $M$ (that is, $M$ collapses onto $P$ ), and when $\# X=0$ (that is, when $M$ is closed) then $P$ is a spine in the usual sense [Matveev 1990], namely $M \backslash\{$ point $\}$ collapses onto $P$. When $\# X \geq 2$, then $M$ does not collapse onto $P$.

Remark 2.1. It is easy to prove that every $(M, X) \in$ $X$ has a skeleton: take any simple spine $Q$ of $M \backslash$ \{point\}, so that $M \backslash Q=\partial M \times[0,1) \cup B^{3}$, and assume that the various $\theta_{i} \times[0,1)$ 's are incident in a generic way to $Q$ and to each other (here of course the $\theta_{i}$ 's are the triods in $X$ ). Taking the union of $Q$ with the $\theta_{i} \times[0,1)$ 's we get a simple $Q^{\prime}$ such that $M \backslash\left(Q^{\prime} \cup \partial M\right)$ consists of $\# X+1$ balls. Then we get a skeleton of $(M, X)$ by puncturing $\# X$ suitably chosen 2-discs embedded in $Q^{\prime}$, so to get one ball only in the complement.

Remark 2.2. A definition of skeleton analogous to our one was given in [Turaev and Viro 1992] for any compact manifold with any trivalent graph in its boundary. The notion of complexity we will now introduce extends to any such object.

We say that a skeleton of $(M, X)$ is nuclear if it does not collapse to a subpolyhedron which is also 
a skeleton of $(M, X)$. We say that a skeleton $P$ of $(M, X) \in \mathcal{X}$ is minimal if it is nuclear and no other skeleton of $(M, X)$ has fewer vertices. We define now the complexity $c(M, X)$ as the number of vertices of any minimal skeleton of $(M, X)$.

Examples with complexity zero.

- It is well-known [Matveev 1990] that the only closed prime manifolds having complexity zero are $S^{3}, S^{2} \times S^{1}, \mathbb{R P}^{3}$, and $L_{3,1}$.

- The trivial element

$$
B_{0}=(T \times[0,1],\{\theta \times\{0\}, \theta \times\{1\}\})
$$

has complexity zero, since it has the simple skeleton $\theta \times[0,1] \subset T \times[0,1]$, which has no vertices.

- Let $H$ be the solid torus, let $D$ be a meridinal disc properly embedded in $H$ and let $\theta \subset \partial H$ be a triod containing $\partial D$, as in Figure 2-left. Then $D \cup \theta$ is a skeleton of $B_{1}=(H,\{\theta\})$, which has therefore complexity zero.
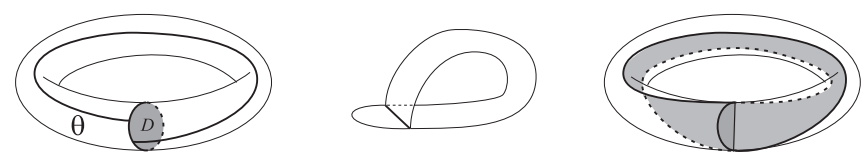

FIGURE 2. The minimal skeleta of $B_{1}$ and $B_{2}$.

- Let $H$ be the solid torus again, and let $P$ be the Möbius strip with one tongue shown in Figure 2centre, embedded in $H$ as in Figure 2-right. Since $P$ has no vertices and it is a skeleton for $B_{2}=$ $(H,\{P \cap \partial H\})$, then $c\left(B_{2}\right)=0$.

\section{B. Properties of Complexity}

Of course we have $c(M, \varnothing)=c(M)$, property (1) of our list from page 209. We prove in this subsection the other properties of $c$. This will require, together with some ad hoc methods, the extension to our context of some techniques used in [Matveev 1990]. In the course of our arguments we will give several definitions used elsewhere in the paper, and we will prove other facts stated above.

Finiteness. The proof of property (5) of complexity requires a careful discussion of the topological properties of minimal skeleta.

A simple polyhedron $Q$ is called quasi-standard if the link of every point is either a circle, or a circle with a diameter, or a circle with three radii (neighbourhoods of points of the three types are shown in Figure 3).
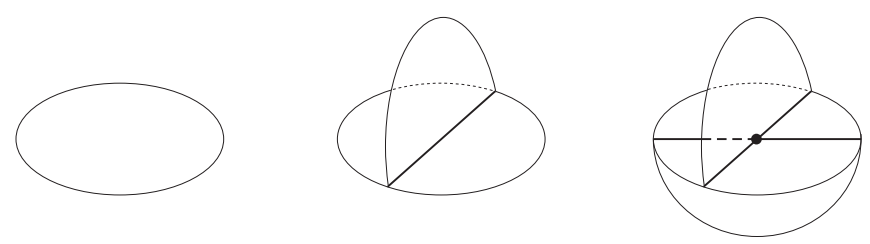

FIGURE 3. Typical neighbourhoods of points in a quasi-standard polyhedron.

A simple polyhedron $Q$ is called quasi-standard with boundary if in addition to these three types of points we have points having as a link either a closed segment or the union of 3 closed segments with one common endpoint. Assuming $Q$ to be quasi-standard with boundary, we denote by $V(Q)$ the set of points (called vertices above) whose link is a circle with three radii, and by $S(Q)$ the union of $V(Q)$ with the set of points whose link is a circle with a diameter. We also denote by $\partial Q$ the points of the two new types declared legal when passing from 'quasi-standard' to 'quasi-standard with boundary.' Moreover, we call 1-components of $Q$ the connected components of $S(Q) \backslash V(Q)$ and 2-components of $Q$ the connected components of $Q \backslash(S(Q) \cup \partial Q)$. If the 2-components of $Q$ are open discs (and hence are called just faces), and the 1-components are open segments (and hence called just edges), then we call $Q$ a standard polyhedron with boundary. For short we will often just call $Q$ a standard polyhedron, and possibly specify that $\partial Q$ should or not be empty.

We state now several easy facts concerning nuclear skeleta, and prove a crucial result concerning minimal skeleta.

Remark 2.3. Let $(M, X)$ be a manifold with triods and let $P$ be a nuclear skeleton of $(M, X)$. Then, up to rearranging the components $T_{1}, \ldots, T_{n}$ of $\partial M$, we have that $P=Q \cup s_{1} \cup \cdots \cup s_{m} \cup K$, where:

1. $Q$ is a quasi-standard polyhedron with boundary $\partial Q \subset X$

2. For $i=1, \ldots, m$ we have that $s_{i} \subset \theta_{i}$ is a segment and $Q \cup s_{i}$ appears near $T_{i}$ precisely as the minimal skeleton of $B_{1}$ appears near $\partial B_{1}$ (see Figure 2-left); for $i>m$ we have $\partial Q \supset \theta_{i}$;

3. $K$ is a graph with $K \cap\left(Q \cup s_{1} \cup \cdots \cup s_{m}\right)$ finite and $K \cap V(Q \cup \partial M)$ empty. 
Remark 2.4. Every $(M, X) \in X$ has a minimal skeleton $P^{\prime}=Q \cup s_{1} \cup \cdots \cup s_{m} \cup K^{\prime}$ as above, where in addition $K^{\prime} \cap \partial M=\varnothing$. This is because, without changing $\# V(P)$, we can take the ends of $K$ lying on $\partial M$ and make them slide over $Q \cup s_{1} \cup \cdots \cup s_{m}$ until they reach $\stackrel{\circ}{M}$. Note that the regular neighbourhood of $\theta_{i} \in X$ in $P^{\prime}$ is now either a product $\theta_{i} \times[0,1]$ or the union of an annulus and a segment, as for $B_{1}$.

Remark 2.5. If $P$ is a nuclear and standard skeleton of $(M, X)$ then it is properly embedded, namely $\partial P=\partial M \cap P=X$, and $P \cup \partial M$ is standard without boundary. Moreover $P \cup \partial M$ is a spine of a manifold bounded by one sphere and some tori, so $\chi(P \cup \partial M)=1$. Knowing that $S(P \cup \partial M)$ is 4 valent and denoting by $F(P)$ the set of faces of $P$, we also see that $\# F(P)-\# V(P)=\# X+1$.

Theorem 2.6. Let $(M, X) \in X$ be prime and let $P$ be a minimal skeleton of $(M, X)$. Then:

1. If $c(M, X)>0$ then $P$ is standard;

2. If $c(M, X)=0$ and $X \neq \varnothing$ then

$$
(M, X) \in\left\{B_{0}, B_{1}, B_{2}\right\},
$$

and $P$ is the skeleton described in Section 2A (which is standard for $B_{0}$ and $B_{2}$ only);

3. If $c(M, X)=0$ and $X=\varnothing$ then

$$
(M, X) \in\left\{S^{3}, S^{2} \times S^{1}, L_{3,1}, \mathbb{R P}^{3}\right\}
$$

and $P$ is not standard.

Proof. Our argument closely follows [Matveev 1990]. We can first rule out the case $(M, X)=\left(S^{2} \times\right.$ $\left.S^{1}, \varnothing\right)$, because for it we only need to show that $P$ is not standard. But a standard polyhedron without boundary must have vertices, while $c\left(S^{2} \times S^{1}, \varnothing\right)=$ 0 . So we proceed assuming that $M$ is irreducible.

We will now prove that if $P$ is not standard then $(M, X) \in\left\{B_{1}, S^{3}, L_{3,1}, \mathbb{R P}^{3}\right\}$, and that $P$ is as in Section $2 \mathrm{~A}$ when $(M, X)=B_{1}$. To conclude we will later show that if $P$ is standard and $c(M, X)=0$ then $(M, X) \in\left\{B_{0}, B_{2}\right\}$ and $P$ is as prescribed.

Suppose then $P$ is not standard. First, if $P$ is a point then $(M, X)=\left(S^{3}, \varnothing\right)$. Suppose now $P$ has a 1-dimensional part. So, let $e \subset P$ be a segment disjoint from the 2-dimensional part of $P$. If $e \subset \partial M$, looking at the ball $M \backslash(P \cup \partial M)$, we deduce that there is a properly embedded disc in $M$ intersecting
$P$ in a point of $e$. By irreducibility $M$ is then a solid torus, so $(M, X)=B_{1}$ and $P$ is as in Figure 2-left.

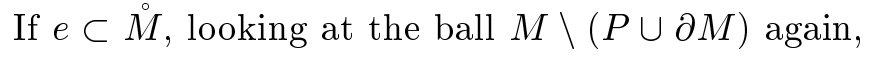
we see that there is a sphere $S \subset M$ intersecting $P$ in one point of $e$. By irreducibility $S$ bounds a ball $B$, and $P \cap B$ is easily seen to be a spine of $B$. Nuclearity now implies that $P \cap B$ contains vertices, so $P \backslash B$ is a skeleton of $(M, X)$ with fewer vertices than $P$. A contradiction.

We have shown so far that $P$ is quasi-standard unless $(M, X)$ is $S^{3}$ or $B_{1}$. Since $P$ is not standard, either a 2-component $f$ is not a disc, or a 1-component is a circle. In the first case, either $f=S^{2}$, or $f=\mathbb{R P}^{2}$, or $f$ contains a simple closed curve $\gamma$ which is nontrivial and orientation-preserving in $f$. In the first two cases we have respectively $P=S^{2}$, which is impossible, and $P=\mathbb{R} P^{2}$, so $(M, X)=\mathbb{R} \mathbb{P}^{3}$. The third case is impossible: looking once more at the ball $M \backslash(P \cup \partial M)$, we deduce that there is a sphere $S \subset M$ intersecting $P$ in $\gamma$, and again $S=\partial B$. As above, $P \cap B$ is a spine of $B$. By minimality $P \cap B$ cannot contain vertices. It follows that $P \cap B$ is a disc, which contradicts the choice of $\gamma$. Finally, if a 1-component of $P$ is a circle but all 2-components are discs, then $P$ must be the "triple hat," a skeleton of $L_{3,1}$.

We are left to analyze the case where $P$ is standard and $c(M, X)=0$, so $X \neq \varnothing$. Now, if $\theta \in X$ and $p$ is a vertex of $\theta$, then the three faces of $P$ incident to $p$ are the same as those incident to the other vertex of $\theta$. Moreover, since $V(P)=\varnothing$, again the same faces are incident to the endpoint of the edge of $P$ which starts at $p$. It easily follows that $F(P) \leq 3$, but $F(P)=1+\# X$ by Remark 2.5 , so $\# X$ is either 1 or 2 . It is now a routine matter to check that $(M, X)$ is respectively $B_{2}$ or $B_{0}$, with $P$ as prescribed.

The next two results show respectively property (5) of complexity and Lemma 1.1. (The first result actually uses also Lemma 3.1 below.)

Corollary 2.7. For any $n \geq 0$, only finitely many pairs in $X^{\mathrm{pr}}$ have complexity $n$.

Corollary 2.8. $\mathcal{B}_{0}^{0}=\varnothing$ and $\mathcal{B}_{0}=\mathcal{B}_{0}^{1}=\left\{B_{0}, B_{1}, B_{2}\right\}$.

Proof. There are no closed bricks of complexity zero, since $\left(S^{2} \times S^{1}, \varnothing\right),\left(S^{3}, \varnothing\right),\left(\mathbb{R P}^{3}, \varnothing\right)$, and $\left(L_{3,1}, \varnothing\right)$ can be obtained assembling respectively two copies 
of $B_{1}$, two copies of $B_{1}$, one copy of $B_{1}$ and one of $B_{2}$, and two copies of $B_{2}$. Moreover $B_{0}, B_{1}$, and $B_{2}$ are not nontrivial assemblings of each other, and the conclusion follows.

Subadditivity under (self-)assembling. Suppose $(M, X)$ and $\left(M^{\prime}, X^{\prime}\right)$ are two pairs, and let $(N, Y)$ be obtained by assembling them. Let $P$ and $P^{\prime}$ be minimal skeleta respectively of $(M, X)$ and $\left(M^{\prime}, X^{\prime}\right)$. The assembling is defined by an identification $\psi$ : $T_{i} \rightarrow T_{i^{\prime}}^{\prime}$ with $\psi\left(\theta_{i}\right)=\theta_{i^{\prime}}^{\prime}$. Using Remark 2.3 we see that $P \cup_{\psi} P^{\prime}$ is simple, so it is a skeleton of $(N, Y)$, and that no new vertices appear. It follows that $c(N, Y) \leq c(M, X)+c\left(M^{\prime}, X^{\prime}\right)$.

Let $(M, X)$ be a pair and let $(N, Y)$ be obtained from $(M, X)$ via a self-assembling, determined by a map $\psi: T_{i} \rightarrow T_{i^{\prime}}$ such that $\psi\left(\theta_{i}\right)$ intersects transversely $\theta_{i^{\prime}}$ in two points. If $P$ is a minimal skeleton of $(M, X)$ as in Remark 2.4, then $P \cup T_{i} \subset N$ is a skeleton for $(N, X)$. Moreover $P \cup T_{i}$ has at most 6 vertices more than $P$ (2 from the vertices of $\theta_{i}, 2$ from those of $\theta_{i^{\prime}}$, and 2 from $\left.\psi\left(\theta_{i}\right) \cap \theta_{i^{\prime}}\right)$. It follows that $c(N, Y) \leq c(M, X)+6$.

Normal surfaces. Let $(M, X)$ be a manifold with triods and let $P$ be a nuclear skeleton of $(M, X)$. The simple polyhedron $P \cup \partial M$ is now a spine of $M$ with a ball $B \subset M$ removed. Choose a triangulation of $P \cup \partial M$, and let $\xi_{P}$ be the handle decomposition of $M \backslash B$ obtained thickening the triangulation of $P \cup \partial M$, as in [Matveev 1990]. In this paragraph we will study closed normal surfaces in $\xi_{P}$. A connected normal surface $S$ is parallel to the boundary when it is obtained by taking one boundary component and pushing it a bit inside $\xi_{P}$. In our case, we have one such surface for each $T_{i}$, and one for $\partial B$.

Two preliminary results are needed to prove our main statement on normal surfaces. The first one refers to another situation, very often considered below, where a normal surface naturally arises.

Proposition 2.9. Let $(M, X)$ be a manifold with triods and let $Q \subset M$ be a quasi-standard polyhedron with $Q \cap \partial M=\partial Q \subset X$. Assume $M \backslash Q$ has two components $N^{\prime}$ and $N^{\prime \prime}$. Then the faces of $Q$ that separate $N^{\prime}$ from $N^{\prime \prime}$ form a closed orientable surface $\Sigma(Q) \subset Q \subset M$ that cuts $M$ into two components.

Proof. Let $e$ be an edge of $Q$, and let $\left\{f_{1}, f_{2}, f_{3}\right\}$ be the triple of (possibly not distinct) faces of $Q$ inci- dent to $e$. The number of $f_{i}$ 's that separate $N^{\prime}$ from $N^{\prime \prime}$ is even; it follows that $\Sigma(Q)$ is a surface away from $V(Q) \cup \partial Q$. Let $T_{i}$ be a boundary component of $M$, containing the triod $\theta_{i} \in X$. Since $T_{i} \backslash \theta_{i}$ is a disc, which is adjacent either to $N^{\prime}$ or to $N^{\prime \prime}$ (say $N^{\prime}$ ), then each 2-component of $Q$ incident to $\theta_{i}$ has $N^{\prime}$ on both sides. So $\Sigma(Q)$ is not adjacent to $\partial Q$. Finally, since $\Sigma(Q)$ intersects the link of each vertex either nowhere or in a loop, then $\Sigma(Q)$ is a closed surface.

The surface $\Sigma(Q)$ cuts $M$ in two components (and is thus orientable, since $M$ is) because $N^{\prime}$ and $N^{\prime \prime}$ lie on opposite sides of $\Sigma(Q)$.

Lemma 2.10. Let $P$ be a standard and nuclear skeleton of a pair $(M, X)$. If $\# V(P)>0$ then every face of $P$ is incident to at least one vertex.

Proof. Assume a face $f$ of $P$ contains no vertices, and let $f$ be incident to the triods $\theta_{i_{1}}, \ldots, \theta_{i_{k}}$. Then $\partial f \cup \theta_{i_{1}} \cup \cdots \cup \theta_{i_{k}}$ is a connected component of $S(P \cup$ $\partial M)$, but $P \cup \partial M$ is standard without boundary by Remark 2.5, so $S(P \cup \partial M)=\partial f \cup \theta_{i_{1}} \cup \cdots \cup \theta_{i_{k}}$, whence $S(P) \subset \partial f$ and $V(P)=\varnothing$. A contradiction.

We go back now to the situation where $P$ is a nuclear skeleton of $(M, X)$.

Lemma 2.11. Let $F$ be a closed normal surface in $\xi_{P}$. Assume that no component of $F$ is boundaryparallel. Then there exists a simple polyhedron $P_{F}$ embedded in $M$, with $\# V\left(P_{F}\right) \leq \# V(P)$, such that $P_{F} \cap \partial M=X$ and $M \backslash\left(P_{F} \cup \partial M\right)$ is an open regular neighbourhood of $F$. Moreover, if $P$ is standard and $\# V(P)>0$ then $\# V\left(P_{F}\right)<\# V(P)$.

Proof. Being normal, $F$ is determined by an integer attached to each 2-component of $P \cup \partial M$. Now we cut $P \cup \partial M$ open along $F$ as explained in [Matveev 1990]: if a 2-component bears an integer $n$ we replace the component by $n+1$ parallel ones. We get a polyhedron $P^{\prime} \subset M$ which contains $\partial M$, such that $M \backslash P^{\prime}$ is the disjoint union of an open ball $B$ and an open regular neighbourhood $N$ of $F$ in $M$. By removing from each torus $T_{i} \subset \partial M$ the open disc $T_{i} \backslash \theta_{i}$ we get a polyhedron $P^{\prime \prime}$ intersecting $\partial M$ in $X$. Now we puncture a 2-component which separates $B$ from $N$ and claim that the polyhedron $P_{F}$ is as desired. Only the inequalities between $V(P)$ and $V\left(P_{F}\right)$ are nonobvious. 
By construction we have $\# V\left(P_{F} \cup \partial M\right) \leq \# V(P \cup$ $\partial M)$. Consider now a vertex $v$ of $P \cup \partial M$ contained in $T_{i} \subset \partial M$. Of the six germs of 2-component of $P \cup \partial M$ at $v$, three are actually the same $T_{i} \backslash \theta_{i}$, so their coefficient in $F$ is the same, say $\alpha$. Call $\beta, \gamma$, and $\delta$ the coefficients of the other three germs of 2component at $v$. As we cut $P \cup \partial M$ along $F$ we see that $v$ disappears if and only if (up to permutation) $\beta=\gamma>\delta$. If $v$ does not disappear then $\beta=\gamma=\delta$ is even. Then we set $k=\alpha-\beta / 2$ and note that $v$ remains on $\partial M$ if and only if $k=0$. Now let $v^{\prime}$ be the other vertex of $P \cup \partial M$ on $T_{i}$. Since the coefficients $(\alpha, \alpha, \alpha, \beta, \gamma, \delta)$ are the same at $v^{\prime}$, we deduce that either $v$ and $v^{\prime}$ both disappear, or they both stay on $\partial M$, or they both move to $\stackrel{M}{M}$. In the last case, however, one sees that $F$ has $k$ components parallel to $T_{i}$, which is absurd. So both $v$ and $v^{\prime}$ disappear in $P^{\prime \prime}$ (either already in $P^{\prime}$ or when we remove $\left.T_{i} \backslash \theta_{i}\right)$. This shows that $\# V\left(P^{\prime \prime}\right) \leq \# V(P)$, so $\# V\left(P_{F}\right) \leq \# V(P)$.

Now suppose that $P$ is standard. Then $P^{\prime \prime}$ is the union of a quasi-standard polyhedron $P^{\prime \prime \prime}$ and some arcs in $X$. The 2-components of $P^{\prime \prime}$ which separate $B$ from $N$ are the same as those of $P^{\prime \prime \prime}$, so they give a closed surface $\Sigma \subset P^{\prime \prime}$ by Proposition 2.9. Since no component of $F$ is parallel to $\partial B$ or to one of the $T_{i}$ 's, the 2-component $f$ of $P^{\prime \prime}$ punctured to get $P_{F}$ cannot be a closed surface. Now if $\partial f$ contains vertices of $P^{\prime \prime}$, we see that $\# V\left(P_{F}\right)<\# V\left(P^{\prime \prime}\right) \leq \# V(P)$, whence the conclusion. Suppose on the contrary that $\partial f$ contains a circle $\gamma \subset S\left(P^{\prime \prime}\right)$ with $\gamma \cap V\left(P^{\prime \prime}\right)=\varnothing$. The process of cutting $P \cup \partial M$ along $F$ allows us to define a local injection $\psi: P^{\prime} \rightarrow P \cup \partial M$, and that $P^{\prime \prime} \subset P^{\prime}$. Now, if $\psi(\gamma)$ contains some vertex of $P$ then this vertex has disappeared in the passage from $P$ to $P^{\prime \prime}$, whence the conclusion. If $\psi(\gamma) \cap V(P)=\varnothing$ then we consider the 2-component $g$ of $P^{\prime \prime} \backslash \Sigma$ incident to $\gamma$ and note that $\psi(g)$ must be a face of $P$ without vertices, which is absurd by Lemma 2.10.

Theorem 2.12. If $(M, X) \in X$ has a standard minimal skeleton, it is prime.

Proof. For $c(M, X)=0$ it was shown during the proof of Theorem 2.6 that $(M, X)$ is $B_{0}$ or $B_{2}$, so we suppose $c(M, X)>0$. By contradiction, assume $M$ is not prime and let $P$ be a standard minimal skeleton of $(M, X)$. Then $\xi_{P}$ contains an essential normal sphere $S$. Such a sphere cannot be parallel to the boundary in $\xi_{P}$. Applying Lemma 2.11 we get $P_{S} \subset M$ with $\# V\left(P_{S}\right)<\# V(P), P_{S} \cap \partial M=$ $X$, and $M \backslash\left(P_{S} \cup \partial M\right) \cong S \times(0,1)$. Since $(S \backslash$ \{point $\}) \times(0,1)$ is an open 3 -ball, adding to $P_{S}$ a generic segment isotopic to $\{$ point $\} \times(0,1)$ we get a skeleton for $(M, X)$ with as many vertices as $P_{S}$. This contradicts minimality of $P$.

Additivity under connected sum. Again we follow [Matveev 1990] quite closely. Let $(M, X)$ and $\left(M^{\prime}, X^{\prime}\right)$ be manifolds with triods, and set $(N, Y)=(M, X) \#$ $\left(M^{\prime}, X^{\prime}\right)$. Let $P$ and $P^{\prime}$ be skeleta of $(M, X)$ and $\left(M^{\prime}, X^{\prime}\right)$, respectively. If we take points $p \in P$ and $p^{\prime} \in P^{\prime}$ which are not vertices and we join them with a segment, we get a skeleton of $(N, Y)$. This implies that $c(N, Y) \leq c(M, X)+c\left(M^{\prime}, X^{\prime}\right)$.

We prove the opposite inequality. Let $P$ be a minimal skeleton of $(N, Y)$. Since $(N, Y)$ is not prime, there is an essential normal sphere $S$ in $\xi_{P}$. We assume first that $S$ is separating. Let $\left(N_{1}, Y_{1}\right)$ and $\left(N_{2}, Y_{2}\right)$ be obtained by cutting $(N, Y)$ along $S$ and gluing in balls. The polyhedron $P_{S}$ given by Lemma 2.11 is now the disjoint union of two polyhedra $P_{1}$ and $P_{2}$ such that $P_{i}$ is a skeleton of $\left(N_{i}, Y_{i}\right)$. Moreover $\# V\left(P_{S}\right)=\# V\left(P_{1}\right)+\# V\left(P_{2}\right) \leq$ $\# V(P)$. Therefore $c\left(N_{1}, Y_{1}\right)+c\left(N_{2}, Y_{2}\right) \leq c(N, Y)$, whence $c\left(N_{1}, Y_{1}\right)+c\left(N_{2}, Y_{2}\right)=c(N, Y)$. If $S$ is non-separating we again consider $P_{S}$ and puncture a suitable region, deducing a decomposition $(N, Y)=$ $\left(N_{1}, Y_{1}\right) \#\left(S^{2} \times S^{1}, \varnothing\right)$ on which complexity is additive. We can now go on finding essential spheres, and additivity eventually follows from uniqueness of the decomposition into primes.

Sharp (self-)assemblings. We are now in a position to prove the second half of properties (3) and (4) of complexity. The case of self-assembling is actually easier, so we start from it. Let a sharp $(N, Y)=$ $\odot(M, X)$ be performed along $\psi: T_{i} \rightarrow T_{i^{\prime}}$. Let $P$ be a minimal skeleton of $(M, X)$ as in Remark 2.4. Then $P \cup T_{i}$ is a minimal skeleton of $(N, Y)$, and it is easy to see that $P$ is standard if and only if $P \cup T_{i}$ is. Moreover, by Theorem 2.6 and Theorem 2.12, $P$ is standard if and only if $(M, X)$ is prime (because $\# X \geq 2)$ and $P \cup T_{i}$ is standard if and only if $(N, Y)$ is prime (because $c(N, Y)>0$ ). This shows the desired conclusion that $(M, X)$ is prime if and only if $(N, Y)$ is. 
To deal with assembling, we need two preliminary results. The first one, together with Theorem 2.6, implies Proposition 1.4.

Lemma 2.13. Let $(M, X) \in X$ be prime and assume $c(M, X)>0$. Then no assembling $(M, X) \oplus B_{1}$ is sharp.

Proof. Let $P$ be a minimal skeleton for $(M, X)$, which is standard by Theorem 2.6, and let $P^{\prime}$ be the minimal skeleton of $B_{1}$. Then $P \cup_{\psi} P^{\prime}$ is a skeleton for $(M, X) \oplus B_{1}$ with minimal number of vertices, but $P \cup_{\psi} P^{\prime}$ is not nuclear: there is a face $f$ of $P$, glued to the free segment of $P^{\prime}$, which is incident to some vertex of $P$ by Lemma 2.10. By collapsing $f$ we would get a skeleton with fewer vertices, which is absurd.

Lemma 2.14. Let $P$ be a minimal skeleton of $(M, X) \in$ $X^{\mathrm{pr}}$ with $c(M, X)>0$. Then, for each $\theta_{i} \in X$, the three faces of $P$ incident to $\theta_{i}$ are distinct from each other.

Proof. By Theorem 2.6, $P$ is standard. Suppose a face $f$ is incident more than once to some $\theta_{i}$. Let $\alpha$ be an arc in $f$ having endpoints $p_{0}$ and $p_{1}$ in two distinct edges of $\theta_{i}$. Of the two essential closed curves $\beta$ such that $\beta \cap \theta_{i}=\left\{p_{0}, p_{1}\right\}$ we choose the one for which a regular neighbourhood of $\alpha \cup \beta$ in $P$ does not contain Möbius strips. Now $\beta$ is cut by $\left\{p_{0}, p_{1}\right\}$ into components $\beta_{0}$ and $\beta_{1}$. Since $M \backslash(P \cup \partial M)$ is a ball, we can glue to both curves $\alpha \cup \beta_{i}$ a disc, and the two discs together form a disc $D \subset M$ with $\partial D=\beta$. Since $\beta$ is essential, $M$ is a solid torus and $(M, X)=B_{1}$.

Now let $(N, Y)=(M, X) \oplus\left(M^{\prime}, X^{\prime}\right)$ be a sharp assembling along some map $\psi: T_{i} \rightarrow T_{i^{\prime}}^{\prime}$. Recall that we want to show that $(N, Y)$ is prime if and only if both $(M, X)$ and $\left(M^{\prime}, X^{\prime}\right)$ are. If $(N, Y)$ is prime, we consider the prime factorization of $(M, X)$ and $\left(M^{\prime}, X^{\prime}\right)$, and note that $\psi$ assembles one factor $W$ of $(M, X)$ to one factor $W^{\prime}$ of $\left(M^{\prime}, X^{\prime}\right)$. If $W \oplus$ $W^{\prime} \neq\left(S^{3}, \varnothing\right)$, then, since $(N, Y)$ is prime, $(M, X)=$ $W$ and $\left(M^{\prime}, X^{\prime}\right)=W^{\prime}$, and we are done. Otherwise, up to permutation, $(M, X)=Z \# W$ and $\left(M^{\prime}, X^{\prime}\right)=$ $W^{\prime}$. By additivity of $c$ under \# and Theorem 2.6, $W$ and $W^{\prime}$ are solid tori, and the assembling is trivial.

Now let $(M, X)$ and $\left(M^{\prime}, X^{\prime}\right)$ be prime. If $c(N, Y)$ vanishes then $c(M, X)=c\left(M^{\prime}, X^{\prime}\right)=0$, so $(M, X)$ and $\left(M^{\prime}, X^{\prime}\right)$ are solid tori by Theorem 2.6. It follows that $(N, Y)$ is a lens space, so it is prime. Assume on the contrary that $c(N, Y)$ is positive. Up to permutation, $c(M, X)>0$. Let $P$ and $P^{\prime}$ be minimal skeleta of $(M, X)$ and $\left(M^{\prime}, X^{\prime}\right)$ respectively, so $P \cup_{\psi} P^{\prime}$ is a skeleton of $(N, Y)$ with minimal number of vertices. If $\left(M^{\prime}, X^{\prime}\right)=B_{1}$, we get a contradiction to Lemma 2.13. Otherwise Theorem 2.6 implies that $P$ and $P^{\prime}$ are standard. To conclude that $(N, Y)$ is prime, using Theorem 2.12, we only need to show that $P \cup_{\psi} P^{\prime}$ is standard. This is not a priori obvious, because some annular component could appear, but Lemma 2.14 applied to $P$ shows that they actually do not, and our argument is complete.

Remark 2.15. Given $(H,\{\theta\}) \in X$ with $H$ the solid torus, it is easy to see that there are infinitely many $\left(H,\left\{\theta^{\prime}\right\}\right)$ 's such that $(H,\{\theta\}) \oplus\left(H,\left\{\theta^{\prime}\right\}\right)=\left(S^{3}, \varnothing\right)$, so

$$
((M, X) \#(H,\{\theta\})) \oplus\left(H,\left\{\theta^{\prime}\right\}\right)=(M, X)
$$

for any $(M, X)$. However, the only assemblings of this sort on which complexity is additive are those where $c(H,\{\theta\})=c\left(H,\left\{\theta^{\prime}\right\}\right)=0$. This can only happen if $\left\{(H,\{\theta\}),\left(H,\left\{\theta^{\prime}\right\}\right)\right\}$ is either $\left\{B_{1}, B_{1}\right\}$ or $\left\{B_{1}, B_{2}\right\}$, so these are the only cases which our definition of trivial rules out from the notion of sharp assembling.

\section{THE ALGORITHM TO FIND BRICKS}

We will explain in this section how we have been able to determine $\mathcal{B}_{\leq 9}$.

\section{A. Properties of Minimal Skeleta of Bricks}

We will introduce in this subsection two more bricks $B_{3}$ and $B_{4}$, besides the $B_{0}, B_{1}$ and $B_{2}$ already defined above. Then we will state some results giving strong restrictions on the shape of minimal skeleta of bricks different from $B_{0}, \ldots, B_{4}$. Later we will describe the operations which we actually have carried out by computer to determine $\mathcal{B}_{\leq 9}$.

Minimal skeleta for $\mathrm{B}_{3}$ and $\mathrm{B}_{4}$. We define $B_{3}$ and $B_{4}$ as the elements of $X$ based on $D_{1} \times S^{1}$ and $D_{2} \times S^{1}$ respectively, where $D_{i}$ is the disc with $i$ holes, and the boundary triods are as decribed in Table 1 (Section 1B). A skeleton for $B_{3}$ is given by the union of an annulus $D_{1} \times$ \{point $\}$ and a ribbon, glued as 

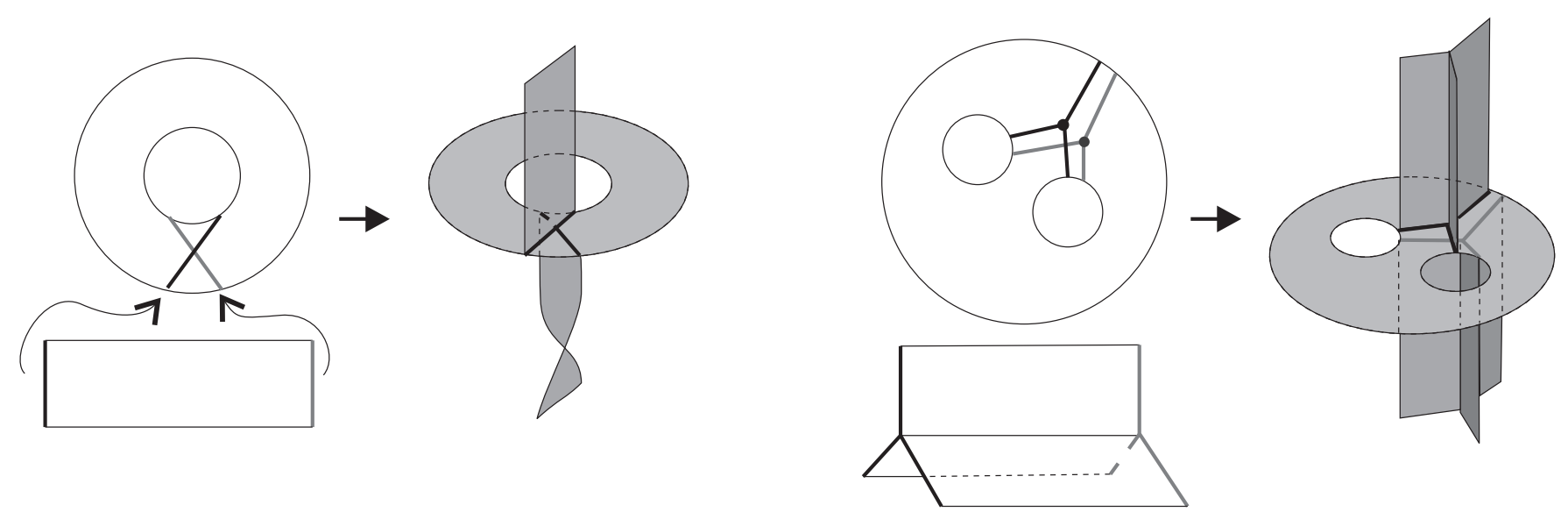

FIGURE 4. Minimal skeleta for $B_{3}$ and $B_{4}$. In the 3-dimensional (gray) pictures the segment and the $Y$ on the top are identified with the corresponding segment and $Y$ on the bottom.

in Figure 4-left. Similarly, a skeleton for $B_{4}$ is given by the union of $D_{2} \times\{$ point $\}$ and a polyhedron as in Figure 4-right, glued as shown. This implies that $c\left(B_{3}\right) \leq 1$ and $c\left(B_{4}\right) \leq 3$. Since $B_{3}$ is prime and it is not $B_{0}, B_{1}$, or $B_{2}$, we have $c\left(B_{3}\right)=1$ by Theorem 2.6-(2). Using Theorem 2.6-(1) and checking by hand all standard $P$ 's with $\# V(P)=1$ and $\partial P \neq \varnothing$, we see that $B_{3}$ is a brick and actually $\mathcal{B}_{1}^{1}=\left\{B_{3}\right\}$. For $B_{4}$ we need:

Lemma 3.1. Let $(M, X)$ be prime and different from $B_{0}, \ldots, B_{3}$. Then $c(M, X) \geq \# X$.

Proof. Of course we can assume $X \neq \varnothing$. Since $\mathcal{B}_{\leq 1}^{1}=\left\{B_{0}, \ldots, B_{3}\right\}$ and the inequality is easy for any nontrivial assembling of $B_{0}, \ldots, B_{3}$, we also assume $c(M, X) \geq 2$.

Suppose now that a face $f$ is incident to two distinct triods $\theta_{i}, \theta_{i^{\prime}} \in X$. Then there is an $\operatorname{arc} \lambda \subset f$, properly embedded in $f$, with endpoints $p \in \theta_{i}, p^{\prime} \in$ $\theta_{i^{\prime}}$, and two essential loops $\gamma \subset T_{i}$ and $\gamma^{\prime} \subset T_{i^{\prime}}$ such that $\gamma \cap \theta_{i}=\{p\}, \gamma^{\prime} \cap \theta_{i^{\prime}}=\left\{p^{\prime}\right\}$. Since $M \backslash(P \cup \partial M)$ is a ball, there is an annulus $A$ properly embedded in $M$, with $\partial A=\gamma \cup \gamma^{\prime}$ and $A \cap P=\lambda$. If some face $g \neq f$ is incident to the same $\theta_{i}$ and to some other $\theta_{i^{\prime \prime}}$, we can construct an annulus $B$ in the same way. Moreover $\partial B=\delta \cup \delta^{\prime \prime}$ with $\#(\gamma \pitchfork \delta)=1$. Irreducibility allows to assume that $A \cap B$ is just one segment, hence $\theta_{i^{\prime}}=\theta_{i^{\prime \prime}}$, and then to show that $M=T \times[0,1]$. So $\# X=2$, but we are assuming $c(M, X) \geq 2$, and the conclusion holds in this case.

By Lemma $2.14, P$ has distinct faces $f_{i}^{(1)}, f_{i}^{(2)}, f_{i}^{(3)}$ incident to each $\theta_{i}$. By what already shown we can assume up to permutation that $f_{i}^{(2)}$ and $f_{i}^{(3)}$ are not incident to any other triod in $X$. So $P$ contains at least $2 \# X+1$ distinct faces. By Remark 2.5, $\# F(P)=\# V(P)+\# X+1$, so $\# V(P) \geq \# X$.

Proposition 3.2. $c\left(B_{4}\right)=3$ and $B_{4}$ is a brick.

Proof. $B_{4}$ is prime and $\# X\left(B_{4}\right)=3$, so $c\left(B_{4}\right)=3$ by the previous lemma. If $B_{4}$ were not a brick then it would split as $B_{i} \oplus B^{(1)} \oplus \cdots \oplus B^{(k)}$ with $i \in\{1,2,3\}$. In all cases we must have $\# X\left(B^{(j)}\right)>c\left(B^{(j)}\right)$ for some $j$, which contradicts the previous lemma.

Superstandard skeleta. A standard polyhedron $P$ (with boundary) is called superstandard if every face of $P$ is incident to $\partial P$ along one segment at most. For such a $P$, it is easy to prove that $S(P)$ must be connected if $P$ is. The minimal skeleta of $B_{0}, \ldots, B_{4}$ we have already described are not superstandard. The following theorem will be proved in Section 4 .

Theorem 3.3. Let $(M, X)$ be a brick different from $B_{0}, \ldots, B_{4}$. Then every minimal skeleton of $(M, X)$ is superstandard.

Corollary 3.4. Let $(M, X)$ be a brick different from $B_{0}, \ldots, B_{4}$. Then $c(M, X) \geq 2 \# X-1$.

Proof. Let $P$ be a minimal skeleton of $(M, X)$. By Remark 2.5 we have $\# F(P)-\# V(P)=\# X+1$. Now $P$ is superstandard, so each edge in $X$ determines a different face of $P$. Then $\# F(P) \geq 3 \# X$, and the conclusion follows.

Enumeration of bricks. Let $(M, X)$ be a brick different from $B_{0}, \ldots, B_{4}$, and let $P$ be one of its minimal skeleta. We will call a filling of $P$ any of the (finitely many) polyhedra obtained by glueing to $P$ 
one copy of the Möbius strip with one tongue along each of the boundary triods of $P$ (so $\# X$ strips in all are glued to $P$ ). Since the Möbius strip with one tongue is a skeleton of the pair $B_{2}$ based on the solid torus, a filling of $P$ is automatically a skeleton of a (possibly nonsharp) assembling of $(M, X)$ with $\# X$ copies of $B_{2}$, hence of a closed manifold $(N, \varnothing) \in \mathcal{X}$ obtained by Dehn-filling all boundary components of $M$. The glueing function $\psi$ used to define the filling of one component $T_{i}$ of $\partial M$ must map the triod $\theta_{i} \subset T_{i}$ to the triod of $B_{2}$, so indeed there are finitely many possibilities. Since $P$ is superstandard by Theorem 3.3, it is easy to see that the fillings of $P$ are standard.

We will call a loop in $P$ a subpolyhedron $\gamma \subset P$ homeomorphic to $S^{1}$ which intersects $S(P)$ transversely (in particular $\gamma \cap V(P)=\varnothing$ ). The length $l(\gamma)$ of $\gamma$ is the number of its intersections with the edges of $P$. We denote by $\mathcal{R}(\gamma)$ a regular neighbourhood of $\gamma$ in $P$. The core of the Möbius strip has length 1 in the Möbius strip with one tongue. The following result will be shown in Section 4 .

Theorem 3.5. Let $(M, X)$ be a brick different from $B_{0}, \ldots, B_{4}$. Let $P$ be a minimal skeleton of $(M, X)$ and let $Q$ be any filling of $P$. Let $\mathcal{L}(Q)$ be any set of representatives of the ambient isotopy classes of length-1 loops in $Q$. Then:

1. The elements of $\mathcal{L}(Q)$ are pairwise disjoint, and $\mathcal{R}(\gamma)$ is a Möbius strip with one tongue for all $\gamma \in \mathcal{L}(Q)$

2. $\mathcal{L}(Q)$ consists of \#X loops and $P=Q \backslash \mathcal{R}(\mathcal{L}(Q))$.

Remark 3.6. The theorem's conclusion (1) implies:

- for every edge $e$ of $Q$, there is no face $f$ of $Q$ triply incident to $e$;

- if $f$ is doubly incident to $e$ and $\partial f$ is given any orientation, then $e$ is induced the same orientation twice;

- if $f_{1}$ is doubly incident to $e_{1}$ and $f_{2}$ is doubly incident to $e_{2}$, with $e_{1} \neq e_{2}$, then $f_{1} \neq f_{2}$.

In addition, taking point (2) of Theorem 3.5 for granted, superstandardness of $P$ (stated by Theorem 3.3) means the following:

- with the notation above, for $i=1,2$, let $g_{i}$ be the face other than $f_{i}$ incident to $e_{i}$; then $f_{1}, f_{2}, g_{1}, g_{2}$ are pairwise distinct.
We state now a result on the singular set $S(Q)$ of a filling $Q$ of a minimal skeleton $P$, noting first that $S(Q)$ depends on $P$ only and it is a 4 -valent graph (because $\partial Q=\varnothing$ ). We refer again to Section 4 for the proof.

Theorem 3.7. Let $(M, X)$ be a brick with nonzero complexity. Let $P$ be a minimal skeleton of $(M, X)$, and let $Q$ be a filling of $P$. Then $S(Q)$ is connected and satisfies the following:

1. No pair of edges disconnects $S(Q)$.

Suppose in addition either that every torus in $M$ is separating or that $c(M, X) \leq 9$. Then:

2. If a quadruple of edges disconnects $S(Q)$, then one of the two resulting components must be of one of the forms shown in Figure 5.
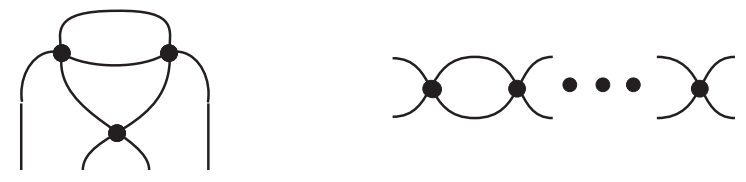

FIGURE 5. If 4 edges disconnect $S(Q)$, then one of the two pieces is of one of these types.

An important tool of our search for bricks is the following nonminimality criterion, proved in Section 3B. We say that a loop $\gamma$ in a skeleton $P$ of $(M, X) \in X$ bounds an external disc if there exists a closed $\operatorname{disc} D \subset M$ with $\partial D=\gamma$ and $D \cap P=\gamma$. A loop is fake if it is contained in the link of some point of $P$.

Theorem 3.8. Let $P$ be a standard skeleton of a manifold with triods. If $P$ contains a non-fake loop which bounds an external disc and has length at most 3, then $P$ is not minimal.

Computer search for bricks. To find $\mathcal{B}_{\leq 9} \backslash\left\{B_{0}, \ldots, B_{4}\right\}$ we have first listed by computer the 4 -valent graphs satisfying the conditions of Theorem 3.7. For each such graph $\Gamma$, using [Benedetti and Petronio 1995], we have then determined the standard spines $Q$ of closed manifolds with $S(Q) \cong \Gamma$ and satisfying the conditions of Remark 3.6. Then we have tested the $Q \backslash \mathcal{R}(\mathcal{L}(Q))$ 's for minimality using Theorem 3.8. The result has been a very short list of skeleta, but actually not all of them were minimal, and some pairs of them were minimal skeleta of the same element of $X$. To eliminate nonminimal and duplicate skeleta we have therefore used certain moves 
on polyhedra which are known to transform a skeleton $P$ into another skeleton $P^{\prime}$ of the same $(M, X)$. Namely, we have used the Matveev-Piergallini move and some disc-replacement moves involving discs of length at most 4 (see [Matveev 1990] for definitions). The result has been a list of minimal skeleta of pairwise distinct elements of $\mathcal{X}^{\text {pr }}$, but a few non-bricks were still present. To get rid of them we have used very technical extra criteria (such as Theorem 4.14 below). The fact that the list of 30 elements eventually obtained cannot be further reduced, so all its elements are indeed bricks, follows from the (easy) fact that no element of the list is obtained via a sharp-assembling from the other ones.

Remark 3.9. The bound $c(M, X) \leq 9$ in Theorem 3.7 is definitely not sharp, and we actually conjecture the theorem to be true for any complexity. Moreover, if an assembling of some bricks is a manifold in which each torus is separating, then the same happens in the individual bricks. Therefore, if one wants to search for closed atoroidal manifolds only, the search for bricks can be restricted to those in which each torus is separating, to which the whole of Theorem 3.7 applies.

We explain now how Theorem 3.7 helps saving space in the search for bricks, by ruling out most graphs as possible $S(Q)$ 's. Namely, let $\mathcal{K}$ be the set of all 4 -valent graphs, let $\mathcal{K}_{\text {brick }} \subset \mathcal{K}$ consist of all $S(Q)$ 's where $Q$ is a filling of some minimal skeleton of some brick, and let $\mathcal{H} \subset \mathcal{K}$ consist of the graphs satisfying both the constraints of Theorem 3.7. We know that $\mathcal{K}_{\text {brick }} \subset \mathcal{H} \subset \mathcal{K}$ (at least in complexity up to 9 , or for bricks in which all tori are separating). Table 3 lists, up to 10 vertices, the number of elements of each of these sets, showing that indeed $\# \mathcal{H}$ is a lot smaller than $\# \mathcal{K}$, and not so far from $\# \mathcal{K}_{\text {brick. }}$. We still have not determined the bricks with 10 vertices.

\section{B. The Nonminimality Criterion}

We prove here Theorem 3.8.

Remark 3.10. Let $(M, X) \in \mathcal{X}$ be given together with a standard skeleton $P$. A closed surface $F \subset P$ contains a graph $H=F \cap S(P)$ with vertices having valency 3 and 4 . If $F$ is orientable, then we can choose a transverse orientation and give each edge $e$ of $H$ a red or black color, depending on whether
$P$ locally lies on the positive or on the negative side of $F$ near $e$. A vertex with valency 3 is adjacent to edges with the same color, and a vertex with valency 4 is adjacent to two opposite red edges and two opposite black ones.

Proof of Theorem 3.8. Consider an external disc $D$ bounded by a loop as in the statement. If we add $D$ and remove a face in $\Sigma(P \cup D)$ we get another skeleton of $P$. We prove now that there is a face in $\Sigma(P \cup D)$ incident to more than $l(\partial D)$ distinct vertices. This shows that $P$ is not minimal.

We consider the graph

$$
H=S(P \cup D) \cap \Sigma(P \cup D),
$$

which contains $\partial D$. By Proposition 2.9, the surface $\Sigma(P \cup D)$ is orientable; we can then choose a transverse orientation and color the edges as explained in Remark 3.10. Suppose by contradiction that each face $f \subset \Sigma(P \cup D)$ meets at most $l(\partial D)$ vertices.

A vertex in $\partial D$ has valency 4 if and only if it is adjacent to two distinct edges in $\partial D$ with distinct colors. If $l(\partial D)=1$, then the only vertex contained in $\partial D$ would have valency 3 , as in Figure 6-(1). So $f_{1}$ would meet at least 2 distinct vertices.

If $l(\partial D)=2$, then the two vertices adjacent to $\partial D$ have the same valency. Suppose they both have valency 4 , as in Figure 6-(2). Since each $f_{i}$ meets at most 2 vertices, then $H$ is as in Figure 6-(3), but $M \backslash(P \cup D)$ would have 3 components. Suppose both vertices of $D$ have valency 3 : then $H$ is as in Figure 6-(4), and $\partial D$ is fake. Both cases are excluded.

If $l(\partial D)=3$, either all vertices met by $\partial D$ have valency 3 , or two of them have valency 4 . Suppose the first case holds. If a face $f_{i}$ meets 2 distinct vertices only, then the other two faces adjacent to $D$ coincide, as in Figure 6-(5), and meet more than 3 vertices. So each $f_{i}$ meets 3 distinct vertices, and $H$ is the 1-skeleton of the tetrahedron $\Sigma(P \cup D)$ as in Figure 6-(6); hence $\partial D$ is fake, which is absurd.

\begin{tabular}{|rrrrrrrrrrr|}
\hline vertices & 1 & 2 & 3 & 4 & 5 & 6 & 7 & 8 & 9 & 10 \\
\hline $\mathcal{K}$ & 1 & 2 & 4 & 10 & 28 & 97 & 359 & 1635 & 8296 & 48432 \\
$\mathcal{H}$ & 1 & 1 & 1 & 2 & 4 & 11 & 27 & 57 & 205 & 1008 \\
$\mathcal{K}_{\text {brick }}$ & 1 & 1 & 1 & 2 & 3 & 1 & 4 & 9 & 13 & $?$ \\
\hline
\end{tabular}

TABLE 3. Graphs which are singular sets of bricks. 


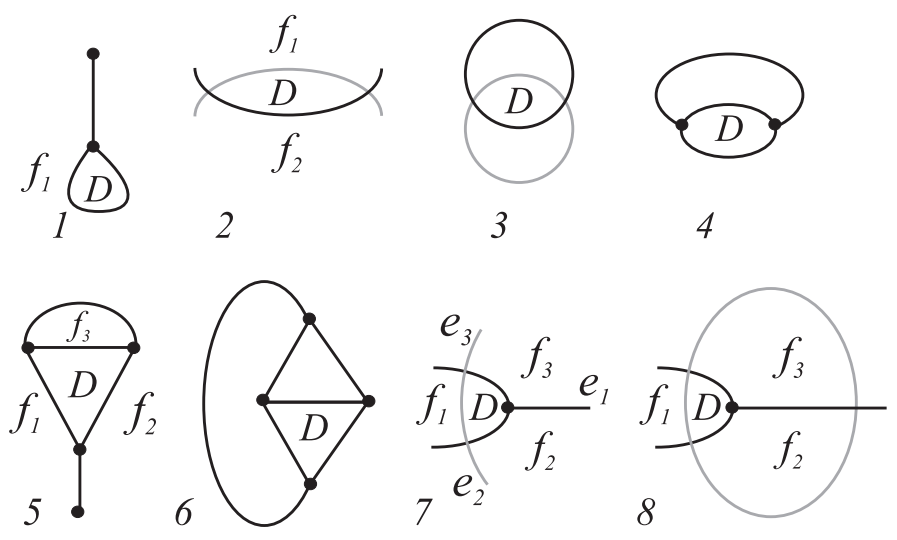

FIGURE 6. Possible configurations for $\Sigma(P \cup D)$ in the proof of Theorem 3.8.

Finally, suppose two vertices have valency 4 and one has valency 3 as in Figure $6-(7)$; since $f_{2}$ is incident to at most 3 distinct vertices, then the distinct edges $e_{1}, e_{2}$ have one common endpoint; for the same reason the edges $e_{1}, e_{3}$ have one common endpoint. Then $H$ is as in Figure 6-(8); but this is absurd since $M \backslash(P \cup D)$ would have at least 3 components.

\section{MINIMAL SKELETA OF BRICKS}

In this section we prove Theorems 3.3, 3.5, and 3.7. This requires the introduction of several ideas not mentioned yet. The crucial point of our work will be the analysis of the intersection between a minimal skeleton and a closed orientable surface. We warn the reader that the proofs of Theorems 4.6 and 4.14 given below are long and not very much illuminating by themselves, so they can be skipped at first. We will only consider in this section bricks having positive complexity, without further notice. So all minimal skeleta will be standard by Theorem 2.6.

\section{A. Traces}

Let $(M, X)$ be a manifold with triods and let $P$ be a standard skeleton of $(M, X)$. A closed surface $F \subset$ $\stackrel{\circ}{M}$ is said to be simply transverse to $P$ if:
1. $F$ is transverse to $P$;

2. The intersection of $F$ with $M \backslash P$ consists of a finite number of discs.

The first condition implies that $Y=P \cap F$ is a finite trivalent graph disjoint from $V(P)$, whose vertices lie precisely at the intersection of $Y$ with the edges of $P$ and appear as in Figure 7-left. Such a graph is called the trace of $F$.

Remark 4.1. Let a trivalent graph $Y \subset P \backslash V(P)$ be given, in such a way that $Y \cap S(P)$ consists of all the vertices of $Y$, each appearing as in Figure 7centre. We show that $Y$ is the trace of an essentially unique simply transverse surface $F \subset M$. First, we can uniquely find a surface $\mathcal{N}(Y)$ with boundary, transverse to $P$, which collapses to $Y$ (see $\mathcal{N}(Y)$ near an edge of $P$ in Figure 7-right). The boundary of $\mathcal{N}(Y)$ consists of a finite number of circles that lie on the boundary of a sub-ball $B^{\prime}$ of $B$. Now we can uniquely glue disjoint discs properly embedded in $B^{\prime}$ to these circles, thus getting the desired closed surface $F$.

\section{B. Traces with 2 Vertices}

Lemma 4.2. Let $(M, X)$ be a brick and let $P$ be a minimal skeleton of $(M, X)$. Let $Y \subset P$ be the trace of an orientable surface $F \subset M$. Then each edge of $Y$ has distinct endpoints.

Proof. Suppose $s$ is an edge of $Y$ with common endpoints; since $F$ is orientable, the regular neighbourhood of $s$ in $F$ is an annulus, so there is a component $D_{0} \subset F \backslash Y$ with $\partial D_{0}=s$. Then $\partial D_{0}$ is a length-1 loop; this is impossible by Theorem 3.8, since length-1 loops are never fake.

Let $P$ be a standard skeleton of some $(M, X) \in X$ and let $\theta_{i} \in X$ be the triod contained in $T_{i} \subset \partial M$. Pushing $\theta_{i}$ a bit inside $\stackrel{\circ}{P}$ we get the trace $Y$ of a torus parallel to $T_{i}$. Therefore we say that such a $Y$ is parallel to the boundary (of $P$ ).
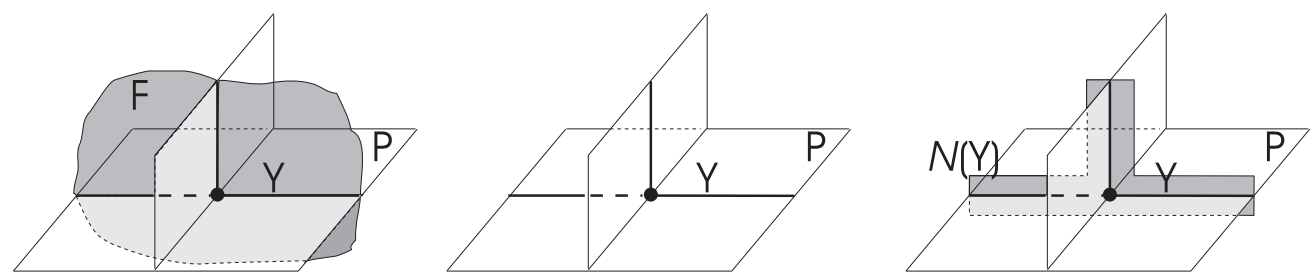

FIGURE 7. A simply transverse surface, its trace, and its reconstruction from the trace. 
Proposition 4.3. Let $(M, X)$ be a brick, equipped with a minimal skeleton $P$. Let $Y$ be the trace of an orientable surface $F$. If $Y$ has two vertices, it is a triod and one of the following cases occurs:

1. $F$ is a nonseparating torus;

2. $Y$ is parallel to the boundary;

3. $F$ is a sphere and $Y$ is the link of a point in $S(P) \backslash V(P)$.

Proof. First, $Y$ is a triod by Lemma 4.2. There are two possibilities for the regular neighbourhood $\mathcal{N}(Y)$ of $Y$ in $F$, which are shown in Figure 8 and lead to a sphere and a torus respectively. In the first case $F \backslash Y$ contains three external discs $D_{i}$ with $e\left(D_{i}\right)=2$. By Theorem 3.8 all the loops $\partial D_{i}$ are fake, so $Y=\operatorname{lk}(p)$ for some $p \in S(P) \backslash V(P)$.
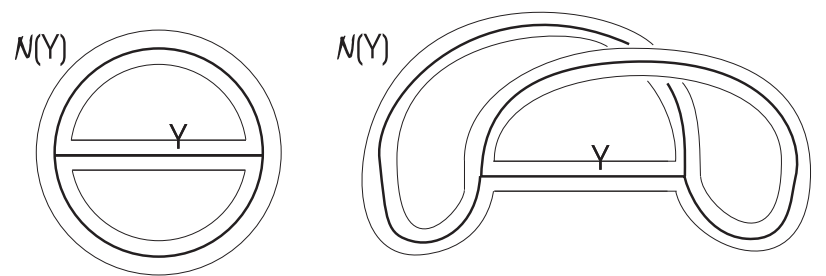

FIGURE 8. The two possibilities for an orientable $\mathcal{N}(Y)$ when $Y$ is a triod.

In the second case, let $F$ be separating, and let $N_{1}$ and $N_{2}$ be the manifolds into which $F$ separates $M$. Set $P_{i}=N_{i} \cap P$ for $i=1,2$. Then $(M, X)$ is obtained by assembling the manifolds with triods $\left(N_{1}, X_{1}\right)$ and $\left(N_{2}, X_{2}\right)$, where $X_{i}=\cup \partial P_{i}$ for $i=1,2$. Moreover $P_{i}$ is a skeleton of $\left(N_{i}, X_{i}\right)$, which implies that this assembling is sharp unless it is trivial. Since $(M, X)$ is a brick, the assembling is trivial. Now, $P_{1}$ and $P_{2}$ are standard, so $\left(M_{1}, X_{1}\right)$ and $\left(M_{2}, X_{2}\right)$ are prime by Theorem 2.12 . Therefore, the assembling must be of the first trivial type, namely $\left(N_{1}, X_{1}\right)$ must be $B_{0}$ up to permutation. Hence $P_{1}$ is the unique minimal skeleton of $B_{0}$, homeomorphic to $\theta \times[0,1]$. It follows that $Y$ is parallel to the boundary in $P$.

Corollary 4.4. Let $P$ be a minimal skeleton of a brick. Then there is no embedded face in $P$ incident to 3 or fewer vertices. Moreover, for every edge e of $P$, the three faces of $P$ incident to $e$ are distinct from each other.

Proof. Let $f$ be an embedded face with 3 or fewer vertices. A loop in $P$ very close to $\partial f$ and disjoint from $\bar{f}$ bounds a disc $D$ parallel to $f$. Moreover $l(\partial D) \leq 3$ and $\partial D$ is not fake since $M \backslash(P \cup \partial M)$ has only one component.

Let $f \subset P$ be a face incident at least twice to an edge $e$ of $P$. It follows that there is a length-1 loop $\gamma \subset P$ intersecting $e$ once. Length-1 loops are never fake, so, by Theorem 3.8, $\gamma$ does not bound a disc. Therefore its regular neighbourhood $\mathcal{R}(\gamma)$ is a Möbius strip with one tongue, and $\partial \mathcal{R}(\gamma)$ is a trace with two vertices of the disconnecting torus in $M$ which bounds the regular neighbourhood of $\gamma$ in $M$. Proposition 4.3 implies that $\partial \mathcal{R}(\gamma)$ is boundaryparallel, so $P$ has no vertices.

Co-disconnecting surfaces. Let $P$ be a standard skeleton of $(M, X) \in X$. Let $Y \subset P$ be the trace of a simply transverse orientable surface $F$. Every component $D$ of $F \backslash Y$ is an open disc; its boundary is the union of two parts $\partial_{1} D$ and $\partial_{2} D$, where $\partial_{i} D$ is the closure of the union of all edges of $Y$ adjacent $i$ times to $D$. If we add $D$ to $P$ we do not get a simple polyhedron, unless $\partial_{2} D=\varnothing$. It is nevertheless easy to see that Proposition 2.9 holds for $P \cup D$ too, namely:

Proposition 4.5. Let $B^{\prime}$ and $B^{\prime \prime}$ be the balls given by $M \backslash(P \cup D)$. The faces of $P \cup D$ that separate $B^{\prime}$ from $B^{\prime \prime}$ form a closed orientable surface $\Sigma(P \cup D) \subset$ $P \cup D \subset M$ which cuts $M$ into two components.

Proof. The proof of Proposition 2.9 works away from $\partial_{2} D$. We only need to show that $\Sigma(P \cup D)$ is a surface near $\partial_{2} D$ : let $f^{\prime}$ and $f^{\prime \prime}$ be the faces other than $D$ incident to an edge $e \subset \partial_{2} D$. Since $F$ is orientable, $f^{\prime}$ is adjacent to $B^{\prime}$ on both sides and $f^{\prime \prime}$ is adjacent to $B^{\prime \prime}$ on both sides (or the converse). Therefore $f^{\prime}$ and $f^{\prime \prime}$ are disjoint from $\Sigma(P \cup D)$, and $\Sigma(P \cup D)$ is a closed surface.

In the setting above we define $\Sigma_{D} \subset P$ as

$$
\Sigma(P \cup D) \backslash D
$$

and call it the co-disconnecting surface of $D$. Proposition 4.5 shows that $\Sigma_{D}$ is a compact surface with boundary $\partial_{1} D$. For a subpolyhedron $K \subset P$ we will denote from now on by $\mathcal{R}(K)$ and $\mathcal{R}_{M}(K)$ the regular neighbourhoods of $K$ in $P$ and in $M$ respectively.

Theorem 4.6. Under the assumptions of Proposition 4.3 , assume that $c(M, X) \leq 9$. Then $Y$ cannot be the trace of a nonseparating torus. 
Proof. By contradiction let $Y=T \cap P$ with $T$ nonseparating, and put $D=T \backslash Y$. The co-disconnecting surface $\Sigma_{D} \subset P$ is by Proposition 4.5 a closed orientable surface, which is nonempty since $\Sigma_{D} \cup T$ disconnects $M$, whereas $T$ does not. We assume that $\#\left(V(P) \cap \Sigma_{D}\right)$ is minimal among all mimimal skeleta of $(M, X)$ for which there exists a nonseparating torus whose trace is a triod.

We focus now on a component $\Sigma$ of $\Sigma_{D}$. Choosing a transverse orientation for $\Sigma$ as in Remark 3.10, we can trace on $\Sigma$ two trivalent graphs $Y_{+}$and $Y_{-}$ which intersect transversely. These graphs represent the way the rest of $P$ glues to $\Sigma$, and the sign + or - depends on whether $P$ locally lies on the positive or on the negative side of $\Sigma$. We show now several properties of the triple $\left(\Sigma, Y_{+}, Y_{-}\right)$which do not require the bound 9 on complexity. Only later will we use this bound.

1. $\Sigma \backslash Y_{ \pm}$consists of planar surfaces. Given a point $p$ of $\Sigma \backslash\left(Y_{+} \cup Y_{-}\right)$there are two points $p_{+}, p_{-}$ of $\partial \mathcal{R}_{M}(P)$ closest to $p$, with $p_{+}$on the positive side of $\Sigma$ and $p_{-}$on the negative side. It is not hard to show that the map $p \mapsto p_{+}$extends to a homeomorphism of $\Sigma \backslash Y_{+}$onto an open subset of $\partial \mathcal{R}_{M}(P) \cong S^{2}$, and similarly for $Y_{-}$.

2. The components of $\partial \mathcal{R}\left(Y_{ \pm}\right) \cap \Sigma$ bound discs in $M$. This follows from the same argument just explained.

3. $\Sigma \backslash\left(Y_{+} \cup Y_{-}\right)$consists of discs. This is because $\Sigma \subset P, Y_{+} \cup Y_{-}=\Sigma \cap S(P)$, and $P$ is standard.

4. If a component of $\Sigma \backslash \mathcal{R}\left(Y_{ \pm}\right)$is not a disc then its boundary loops are essential in $\Sigma$. We refer to $Y_{+}$. If one of them is not, it is very easy to see that there is a disc $\Delta$ in $\Sigma$ such that $Y_{+} \cap \partial \Delta=\varnothing$ but $Y_{+} \cap \Delta \neq \varnothing$, so in particular $Y_{+} \cap \Delta$ contains vertices of $P$. The move suggested in Figure 9 then contradicts minimality of $P$.
5. Not all the components of $\mathcal{R}\left(Y_{ \pm}\right) \cap \Sigma$ are planar. Again we refer to $Y_{+}$. By contradiction, from points 1 and 2 and the irreducibility of $M$, we would readily get that $\Sigma$ bounds a handlebody, but $\Sigma$ is nonseparating.

6. Every component of $Y_{+}$intersects $Y_{-}$, and conversely. Otherwise, since $\Sigma$ is connected, there would exist a component of $\Sigma \backslash\left(Y_{+} \cup Y_{-}\right)$with disconnected boundary, contradicting point 3 .

7. $Y_{+} \cap Y_{-}$contains at least two points. Assume there is only one point $v$ (a crossing between $Y_{+}$ and $Y_{-}$). If a face $f$ of $\Sigma$ is incident to $v$, then it must be multiply incident, because faces contain an even number of quadrivalent vertices (with multiplicity). If two instances of $f$ are adjacent to each other at $v$, we find in the closure of $f$ a length-1 loop bounding an external disc, which contradicts minimality. If two instances of $f$ are opposite at $v$, then for the same reason there is another face $g$ doubly incident to $v$, and $g \neq f$. Now in the closure of $f \cup g$ we can easily find a length-2 loop bounding an external disc which meets edges opposite at $v$. By minimality the loop must be fake, so these edges must actually be the same. Orientability of $\Sigma$ then implies that $f=g$ : a contradiction.

8. A component of $Y_{ \pm}$that is a circle intersects $Y_{\mp}$ in at least 4 points. This readily follows from Corollary 4.4 and minimality, because this circle is precisely the boundary of a face of $P$.

9. There are no squares as in Figure 10-left occurring in $\left(\Sigma, Y_{+}, Y_{-}\right)$. If one such square exists, we can correspondingly apply to $P$ one move as in Figure 10-right. The result is a new minimal skeleton $P^{\prime}$ on which $T$ still has trace $Y$, but $\#\left(V\left(P^{\prime}\right) \cap \Sigma_{D}^{\prime}\right)<\#\left(V(P) \cap \Sigma_{D}\right)$ - a contradiction.

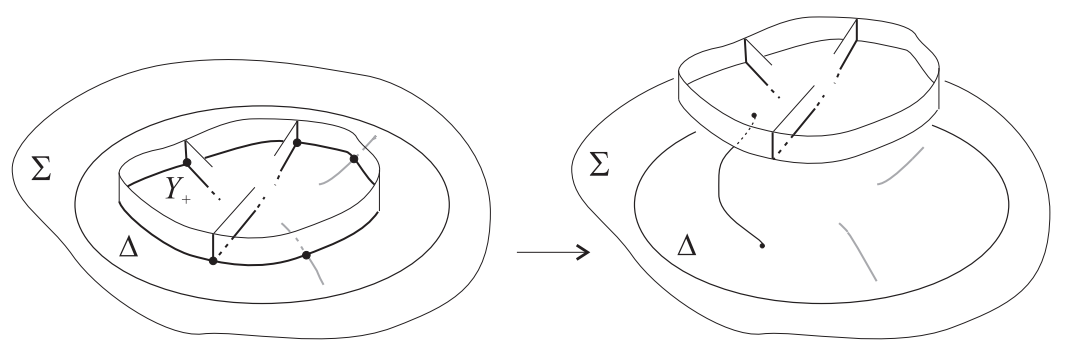

FIGURE 9. A move that reduces complexity. 

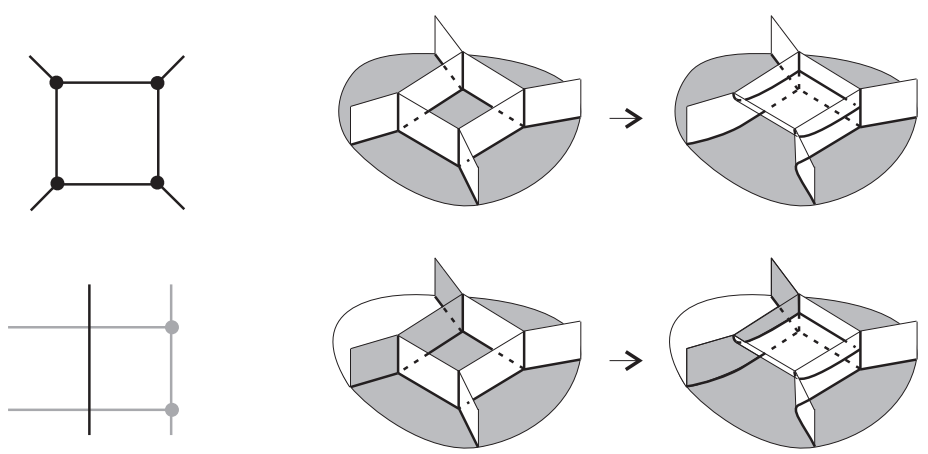

FIGURE 10. Forbidden squares (with black $Y_{+}$and gray $Y_{-}$), and moves (with shadowed $\Sigma$ ).

We show now how to conclude, using the fact that $\# V(P) \leq 9$. It follows from point 5 that both $Y_{+}$ and $Y_{-}$have vertices. Being trivalent, they have an even number of them, and the total is at most $9-2=7$ by point 7 . So up to permutation we can assume that $Y_{+}$has 2 vertices. In particular $\mathcal{R}\left(Y_{+}\right) \cap \Sigma$ has only one non-planar component, which is homeomorphic to a punctured torus (with a component of $Y_{+}$sitting as a triod in this torus). From point 8 we deduce that $Y_{+}$can have at most one circular component, and it is now easy to deduce from point 1 that $\Sigma$ indeed is a torus. Point 4 then implies that $Y_{+}$consists of the triod only. In the rest of our proof we will always depict $\Sigma$ cut open along $Y_{+}$. So $\Sigma$ appears as a hexagon, and we denote by $\Delta$ its interior.

To conclude the proof we will first show that $Y_{-}$ also has 2 vertices, and then that it appears in one of the two shapes shown in Figure 11. This indeed yields a contradiction to the fact that $(M, X)$ is a brick, since $Y_{-} \cap Y_{+}$consists of two points, so cutting $P$ along $\Sigma$ we see that $(M, X)$ can be obtained via a sharp self-assembling.
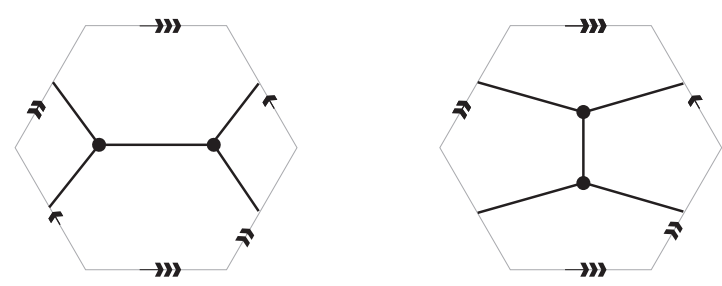

FIGURE 11. Configurations that correspond to a selfassembling.

So, let $Y_{-}$have 4 vertices. We claim that all the components of $Y_{-} \cap \Delta$ are trees. If one of them is not then there is a face of $P$ inside $\Delta$ and bounded by $Y_{-}$. Then either this face has $\leq 3$ vertices, which contradicts Corollary 4.4, or it is a square of the first forbidden type. Our claim is proved. Now note that if $Y_{-} \cap \Delta$ has $\nu$ components then it has $4+2 \nu$ free endpoints, which give $2+\nu$ vertices in $P$. Since $Y_{+}$has 2 vertices and $Y_{-}$has 4 , we deduce that $\nu=1$ and that $Q=P \backslash \mathcal{R}_{P}(\Sigma)$ has no vertices. Moreover $Q$ is connected and standard, and $\partial Q \cong$ $Y_{+} \sqcup Y_{-}$. It is not hard to show that with these constraints the only possibility for $Q$ is as shown in Figure 12, so $\partial \mathcal{R}_{M}(Q)$ has two components. In addition, also $\Sigma \backslash Y_{-}$consists of discs (as $\Sigma \backslash Y_{+}$), and we get a contradiction because $\partial \mathcal{R}_{M}(Q)$ should then be a sphere with some holes.

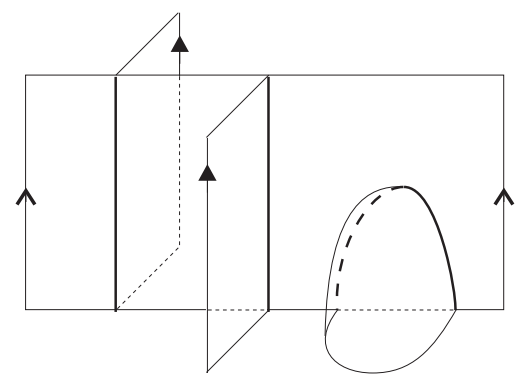

FIGURE 12. A polyhedron without vertices.

We can now assume that $Y_{-}$has two vertices, and show that it appears as in Figure 11. Knowing already that $\Sigma \backslash Y_{-}$is a disc, it is enough to show that $Y_{-} \cap \Delta$ is connected. Suppose by contradiction that $Y_{-} \cap \Delta$ is disconnected. Then there exists an arc $\alpha$ properly embedded in $\Delta$ which separates two components of $Y_{-} \cap \Delta$. Consider the endpoints of $\alpha$. By minimality of $P$, they cannot belong to the same edge of $\Delta$, nor to two adjacent ones, otherwise we could make $Y_{-}$slide on $\Sigma$ and reduce the number of vertices, as in Figure 13.

The ends of $\alpha$ also cannot belong to two edges adjacent to one and the same edge, as in Figure 14left.

To see this, consider how many vertices of $Y_{-}$can lie in $\Delta^{\prime}$. If there are no vertices at all, then either a face of $P$ contained in $\Delta^{\prime}$ has less than 4 vertices or there is a square of the second forbidden type. If $Y_{-}$ has both vertices in $\Delta^{\prime}$, then again $\Delta^{\prime}$ contains either a small face or a forbidden square. These cases are excluded, so there is one vertex of $Y_{-}$in $\Delta^{\prime}$, and the only possible case is shown in Figure 14-center. Now we let $Y_{+}$slide over $\Sigma$ as shown in Figure 14-right. The result is a new minimal skeleton $P^{\prime}$ on which $T$ still has trace $Y$, but $\Sigma_{D}^{\prime}$ now contains one of the 


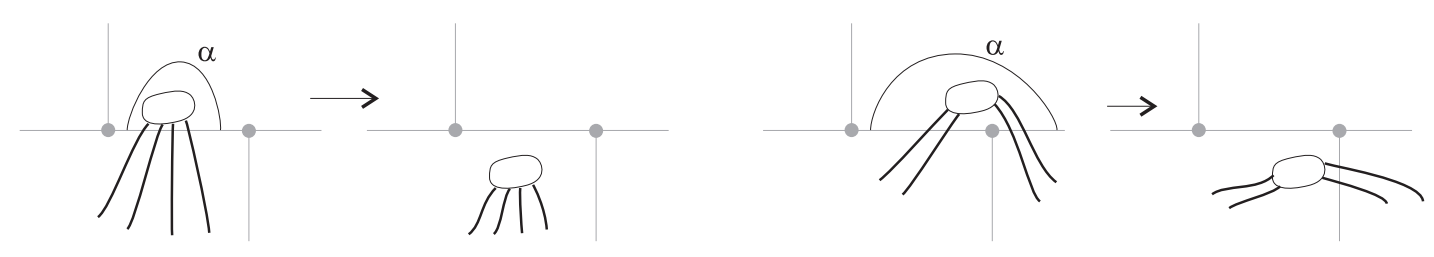

FIGURE 13. Moves reducing complexity.

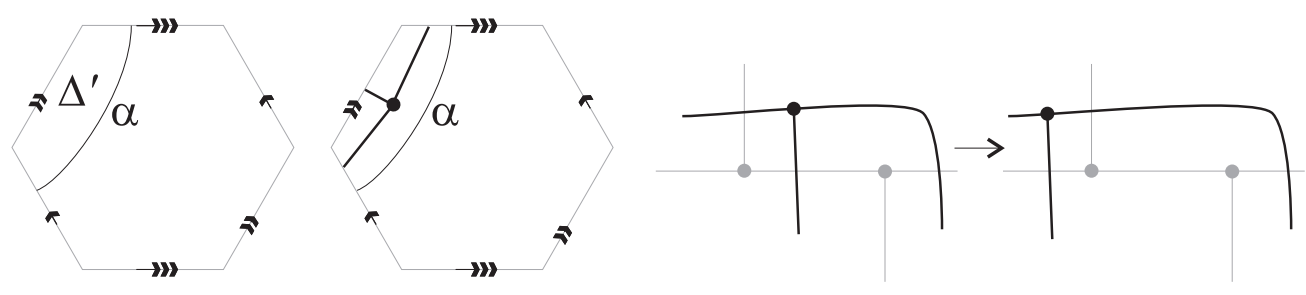

FIGURE 14. More moves reducing complexity.

forbidden squares of Figure 10, which contradicts minimality of $\#\left(V(P) \cap \Sigma_{D}\right)$.

We are left to show that the endpoints of $\alpha$ also cannot belong to opposite edges of $\Delta$ (Figure 15left).

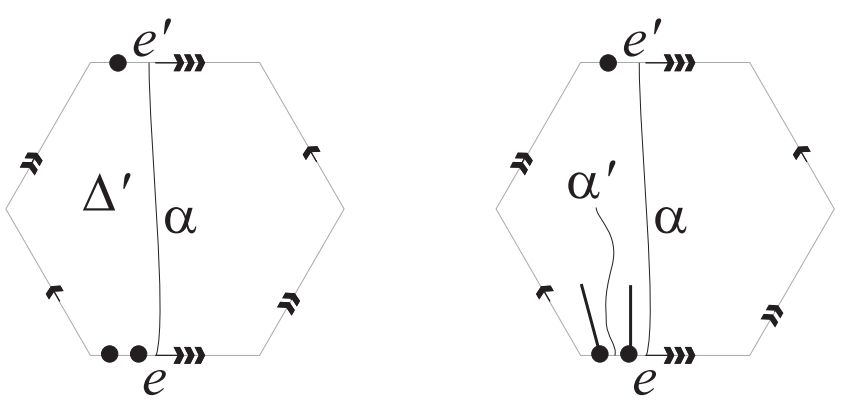

FIGURE 15. Conclusion of Step 3.

Denote by $\nu$ and $\nu^{\prime}$ the number of ends of $Y_{-} \cap \Delta$ on $e \cap \Delta^{\prime}$ and on $e^{\prime} \cap \Delta^{\prime}$ respectively. If $\nu=\nu^{\prime}$ then $\alpha$ can be isotoped so to give rise to a length-1 loop in $P$ bounding an external disc: a contradiction. If $\nu=0$ or $\nu^{\prime}=0$ then we can replace $\alpha$ by a curve disjoint from $Y_{-}$and having ends on edges of $\Delta$ which are not opposite, so we get back to a case already ruled out. So up to permutation we can assume that $\nu \geq 2$. Now the face of $P$ containing the portion of arc $\alpha^{\prime}$ shown in Figure 15-right must meet another edge of $Y_{+}=\partial \Delta$, otherwise it is either small or forbidden (recall that $Y_{-}$has 2 vertices only). So $\alpha^{\prime}$ extends to a properly embedded arc disjoint from $Y_{-}$. Either $\alpha^{\prime}$ belongs to a case already ruled out, or the corresponding $\nu+\nu^{\prime}$ is smaller, and a contradiction is reached anyway. This eventually shows that $Y_{-}$is connected, and the proof is complete.

\section{C. Moves on Traces}

The key step to check the properties of bricks will be Theorem 4.14 stated below. We introduce here more new notions which will be used to prove it.

Let $P$ be a standard skeleton of a manifold with triods $(M, X)$. Given the trace $Y$ of a surface $F$, there are some obvious moves that transform $Y$ into another trace $Y^{\prime}$ of a surface $F^{\prime}$ isotopic to $F$. Three such moves, denoted by $J_{1}, J_{2}$ and $J_{3}$ and collectively called $J$-moves, are shown in Figure 16 . Since we will be concerned with traces of (transversely) orientable surfaces only, we can ask a $J$-move to transform a trace $Y$ into a trace $Y^{\prime}$ disjoint from $Y$. Let $\left[Y, Y^{\prime}\right]$ be the sub-polyhedron which lies between $Y$ and $Y^{\prime}$. A sequence of moves $Y_{1} \rightarrow \cdots \rightarrow Y_{n}$ is called a flow if each move $Y_{i} \rightarrow Y_{i+1}$ is a $J$-move and $\left[Y_{i-1}, Y_{i}\right] \cap\left[Y_{i}, Y_{i+1}\right]=Y_{i}$ for all $i$, namely, if the moves are performed towards the same normal direction to $Y_{i}$ for all $i$.

Remark 4.7. A move $J_{1}$ is determined by an edge $s$ of $Y$ and a vertex $v$ of $P$ such that $s \subset \operatorname{lk}(v)$, or
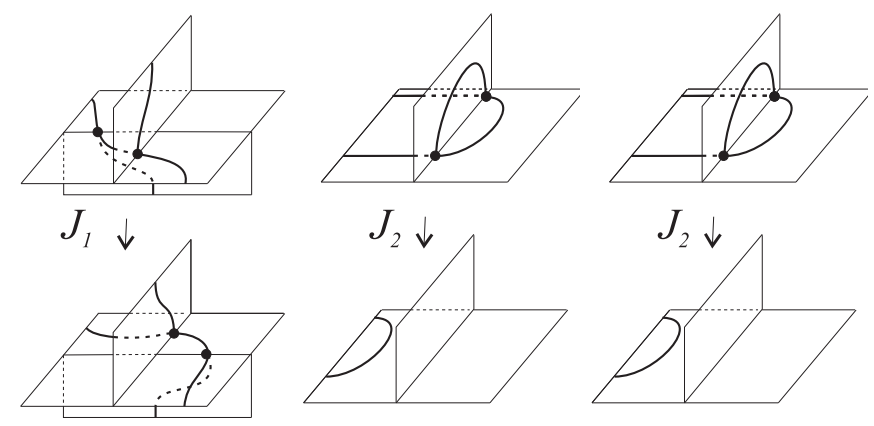

FIGURE 16. The moves $J_{1}, J_{2}, J_{3}$. 
equivalently by the cone $v s$ from $v$ based on $s$ (a triangle). We will sometimes say that the move is performed along the triangle.

Remark 4.8. If a move $J_{1}$ transforms a trace $Y$ of $F$ into a trace $Y^{\prime}$ of $F^{\prime}$ then there is a natural bijection between the components of $F \backslash Y$ and those of $F^{\prime} \backslash Y^{\prime}$.

Let $Y$ be the trace of a surface $F$. Given a component $D$ of $F \backslash Y$, we denote by $e(D)$ the number of edges of $Y$ adjacent to $D$, counted with multiplicity (an edge of $Y$ is counted twice if it has $D$ on both sides).

Lemma 4.9. Let $P$ be a minimal skeleton of a brick. Let $Y \subset P$ be the trace of an orientable surface $F$, and let $D$ be a component of $F \backslash Y$. Consider a move $J_{1}$ determined by an edge $s \subset \partial D$ of $Y$ and a vertex $v$ of $P$, call $Y^{\prime}$ the resulting trace and $D^{\prime}$ the disc corresponding to $D$. Then $e\left(D^{\prime}\right)<e(D)$ if $e(D)<6$ and $e\left(D^{\prime}\right) \leq e(D)$ if $e(D)=6$.

Proof. The trace $Y^{\prime}$ is obtained from $Y$ as shown in Figure 17; it follows from the figure that if $e\left(D^{\prime}\right)>$ $e(D)$ then $D_{1}=D_{2}=D \neq D_{3}$ and if $e\left(D^{\prime}\right)=e(D)$ then $D=D_{1}$ or $D=D_{2}$. By Lemma 4.2 the edges of $Y$ have distinct ends. Using this fact one easily sees that $e(D)>6$ if $D_{1}=D_{2}=D \neq D_{3}$ and $e(D) \geq 6$ if $D=D_{1}$ or $D=D_{2}$, and the conclusion follows.

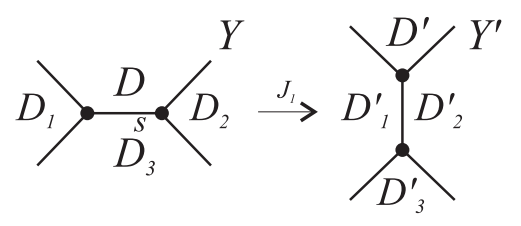

FIGURE 17. A move $J_{1}$ at the level of traces.

Good discs. Let $Y$ be the trace of a surface $F$. We say that a disc $D \subset F \backslash Y$ is good if all discs in $F \backslash Y$ other than $D$ are contained in the same component of $M \backslash(P \cup D)$.

Remark 4.10. If $F$ has 2 discs, these discs are good.

Remark 4.11. If $F$ is orientable, then $\mathcal{R}_{P}(Y) \cong Y \times$ $[-1,1]$. Recall that $\partial \Sigma_{D}=\partial_{1} D$. Now it is not hard to show that if $D$ is good then the identification $\mathcal{R}_{P}(Y) \cong Y \times[-1,1]$ can be chosen so that $\Sigma_{D} \cap$ $\mathcal{R}_{P}(Y) \cong \partial_{1} D \times[0,1]$, and the converse holds if $F$ is connected. In other words, when $F$ is orientable and connected, we have that $D$ is good if and only if $\Sigma_{D}$ lies on a definite side of $Y$ in $P$.

Lemma 4.12. Under the assumptions of Lemma 4.9, suppose that $D$ is good and that $\Sigma_{D}$ and the triangle $v$ s lie on the same side of $Y$ in $P$. Then $D^{\prime}$ is good and $\Sigma_{D^{\prime}}=\Sigma_{D} \backslash\left[Y, Y^{\prime}\right]$.

Proof. The condition that $\Sigma_{D}$ and $s v$ lie on the same of side of $Y$ means that $Y$, during its transformation into $Y^{\prime}$, is pushed towards $\Sigma_{D}$, and the conclusion is obvious.

\section{D. Traces with 4 Vertices}

We prove here the key result needed to establish the properties of bricks.

Remark 4.13. If a polyhedron $P$ is superstandard (with boundary), it can be uniquely reconstructed from the regular neighbourhood $\mathcal{R}(S(P))$ of $S(P)$ in $P$, by gluing discs to each circle in $\partial \mathcal{R}(S(P))$, because the rest of $\partial \mathcal{R}(S(P))$ can be identified to $\partial P$. Therefore here and in the sequel we will describe such $P$ 's by drawing $\partial \mathcal{R}(S(P))$ in $\mathbb{R}^{3}$. Threedimensional pictures will be needed when $P$ is only standard.

Theorem 4.14. Let $P$ be a minimal skeleton of a brick $(M, X)$, and let $Y \subset P$ be a trace with 4 vertices of an orientable connected surface $F$. If $F$ is separating, then $Y$ is a boundary component of a polyhedron of one of the following types:

1. $\mathcal{R}_{P}(v)$ for some $v \in V(P)$ (type 1.1$)$, or $\mathcal{R}_{P}(\lambda)$ for an arc $\lambda$ properly embedded in a face of $P$ (type 1.2);

2. $\mathcal{R}_{P}(\gamma)$ for a length-2 loop $\gamma$, which is fake if it bounds an external disc;

3. one of the 5 polyhedra shown in Figure 18, whose boundary has two components: $Y$ and a triod $\theta_{i} \subset \partial P$;

4. a polyhedron as in Figure 19, with 1 (left) or more (right) vertices.
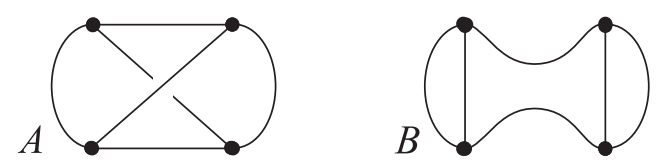

FIGURE 20. Types $A$ and $B$ for $Y$. 

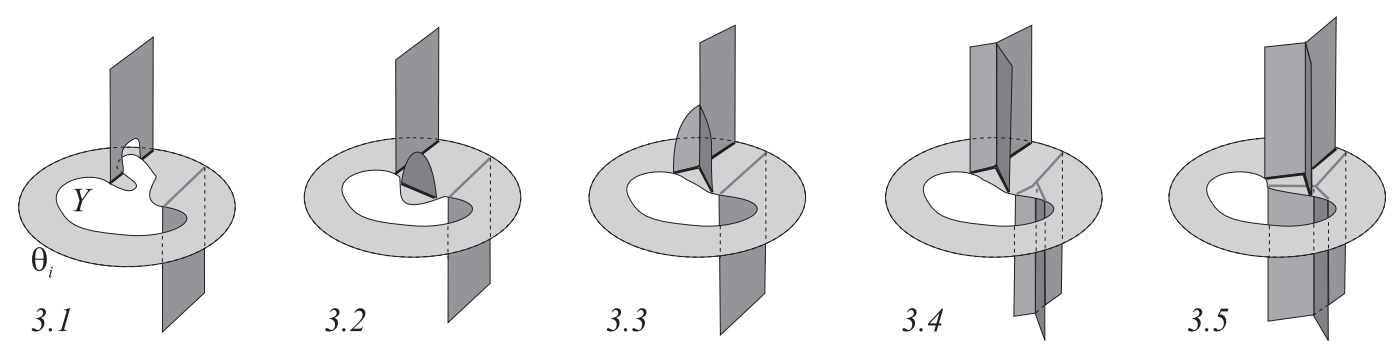

FIGURE 18. Polyhedra of type 3 : segments (in the first 3 pictures) and $Y$ 's on the top are identified to the corresponding ones on the bottom.
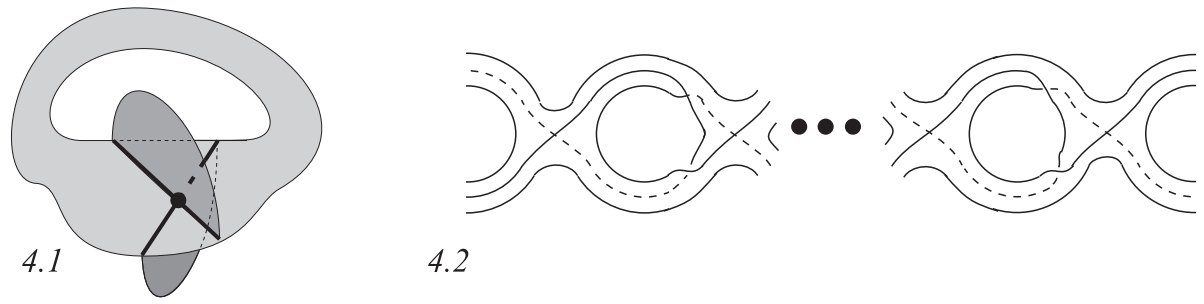

FIGURE 19. Polyhedra of type 4.

If $F$ is not separating, it is a torus and there is a minimal skeleton $P^{\prime}$ of $(M, X)$ on which $F$ has a triod as a trace.

Moreover, only two types $A$ and $B$ of $Y$ are possible, as shown in Figure 20. The polyhedra of types $1.1,3.3$, and 3.4 have boundaries of type $A$, those of types 1.2, 3.1, 3.2, 3.5, and 4 have boundaries of type $B$; a polyhedron of type 2 has boundary of type $A$ if it is a Möbius strip with two tongues, of type $B$ otherwise.

Proof. It is enough to show that one of the following must hold:

(I) $F$ is a nonseparating torus, and $F$ has a triod as a trace on some $P^{\prime}$;

(II) $Y$ bounds one of the polyhedra of type 1-4.
So we assume (I) does not hold and show (II). Our argument is long and organized in many steps. We first describe the overall scheme by stating without proof five assertions. Later we will provide full proofs. Let $D \subset F \backslash Y$ be a component having lowest $e(D)$.

Fact 1. If $e(D) \in\{2,3\}$ then $Y$ bounds a polyhedron of type $1,2,3.1,3.2$, or 3.3 .

Suppose then that $e(D) \geq 4$. Since $Y$ is trivalent it has 6 edges, so $\chi(F)=d-2$, where $d$ is the number of components of $F \backslash Y$. Each component is incident to at least 4 vertices, so $2 \cdot 6 \geq 4 \cdot d$, whence $d \leq 3$. It easily follows that $F$ is a torus and $d=2$. Then $F \backslash Y$ consists of two discs $D=$ $D_{1}$ and $D_{2}$, both good by Remark 4.10. Recalling
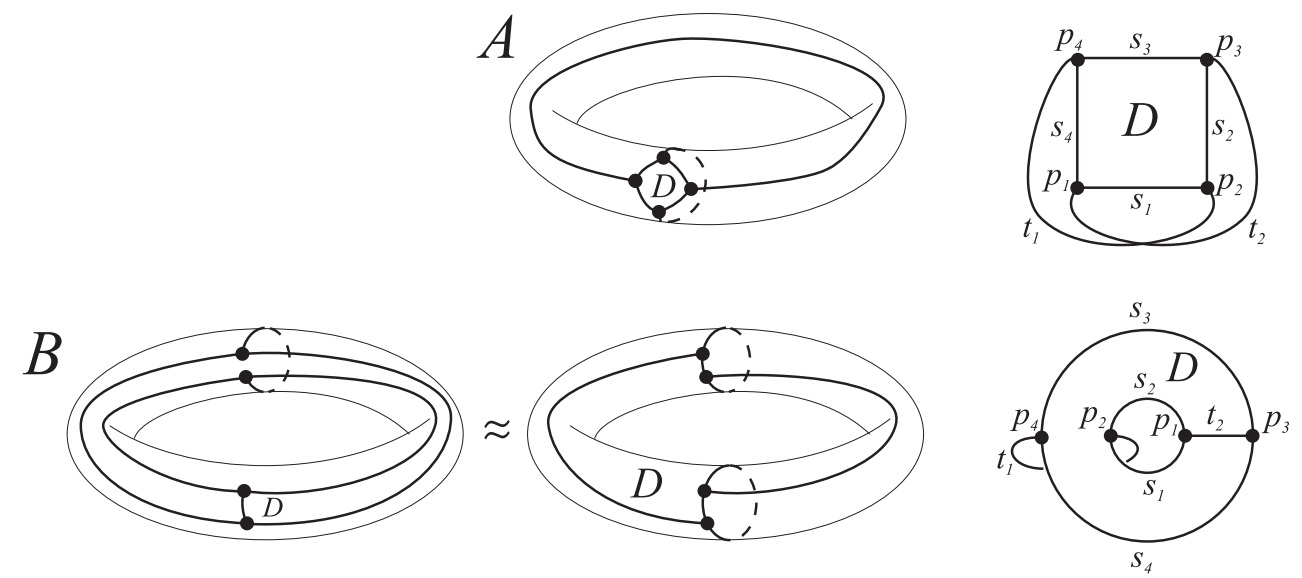

FIGURE 21. Embeddings of type $A$ and $B$. 
from Lemma 4.2 that all edges of $Y$ have distinct endpoints one easily sees that only the types $A$ and $B$ for $Y$ are possible. The restriction that $e(D) \geq$ 4 then implies that up to homeomorphism there is only one possible configuration $\left(F, Y_{A}\right)$ and only one $\left(F, Y_{B}\right)$, as shown in Figure 21. If $Y$ is of type A we have $e(D)=4$, otherwise we have $e(D)=6$, and the two discs of $F$ are completely symmetric.

Figure 21 also contains notation used throughout the proof (note that $s_{1}, \ldots, s_{4}$ are the edges in $\partial_{1} D=\partial \Sigma_{D}$ both in case $A$ and in case $\left.B\right)$. Let $f_{i}$ be the face of $\Sigma_{D} \backslash S(P)$ incident to $s_{i}$. Moreover, let $g_{j}$ be the face of $P$ incident to $t_{j}$. Since $D$ is good, we have $g_{1}, g_{2} \not \subset \Sigma_{D}$. Finally, let $e_{i}$ be the edge of $P$ which contains $p_{i}$.

Fact 2. Either the faces $f_{1}, f_{2}, f_{3}, f_{4}, g_{1}, g_{2}$ are all distinct or $Y$ bounds a polyhedron of type 2 or 4.1.

Assuming that $Y$ does not bound a polyhedron of type 2 or 4.1 , it follows that the segments $e_{i} \cap \Sigma_{D}$ for $i=1, \ldots, 4$ are distinct. Then let $v_{i} \in V(P)$ be the endpoint of $e_{i} \cap \Sigma_{D}$ not lying on $D$.

Fact 3. Up to symmetry we have $v_{1}=v_{2}$ in case $A$ and either $v_{1}=v_{2}$ or $v_{1}=v_{3}$ in case $B$.

Now set $u=s_{1}$ in case $A$, and either $u=s_{1}$ or $u=$ $t_{1}$ in case $B$, depending on whether $v_{1}=v_{2}$ or $v_{1}=$ $v_{3}$, so there are two edges of $P \cup D$ which start at the endpoints of $u$ and both end at $v_{1}$. These edges are $e_{1}^{\prime}=e_{1} \cap \Sigma_{D}$ and $e_{m}^{\prime}=e_{m} \cap \Sigma_{D}$, with $m \in\{2,3\}$ depending on the case. Recall now that if two edges end at the same vertex then one face incident to the first edge is also incident to the second one. Since we are assuming that the $f_{i}$ 's and $g_{j}$ 's are distinct, we deduce that $u \cup e_{1}^{\prime} \cup e_{m}^{\prime}$ bounds a disc of $P \cup D$, which is a triangle; that is, $u \subset \operatorname{lk}\left(v_{1}\right)$. Following Remark 4.7 we can then perform a $J_{1}$-move to which Lemma 4.9 and Lemma 4.12 apply. Denoting by $D^{\prime}$ the disc corresponding to $D$ after the move, we have $e\left(D^{\prime}\right) \leq e(D)$, and equality can hold only if $Y$ is of type $B$.

Fact 4. If $e\left(D^{\prime}\right)<e(D)$ then $Y$ bounds a polyhedron of type 3.4 or 3.5 .

Fact 5. If $e\left(D^{\prime}\right)=e(D)$ then $Y$ bounds a polyhedron of type 4.2 .

Proving these five assertions establishes the theorem; we turn to this task.
Proof of Fact 1. By Theorem 3.8 the loop $\partial D$ is fake, and we can perform a move $J_{e(D)}$ as explained in Section $4 C$. The result is a trace $Y^{\prime}$ with 2 vertices of a surface $F^{\prime}$ isotopic to $F$. By Proposition 4.3 either $F^{\prime}$ is a nonseparating torus, or $Y^{\prime}$ is boundary-parallel, or we have $Y^{\prime}=\partial \mathcal{R}(p)$ for some $p \in V(P) \backslash S(P)$. In the first case, up to isotoping $F^{\prime}$ back to $F$, getting an isotopic copy $P^{\prime}$ of $P$, we get a contradiction to our initial assumption. In the other cases we have to see which polyhedra can result from an inverse $J_{e(D)}$ move applied to $\theta_{i} \times[0,1]$ or to $\mathcal{R}(p)$. It is now rather easy to examine all possibilities and check the assertion.

Proof of Fact 2. Of course no $f_{i}$ can be equal to a $g_{j}$, because $f_{i} \subset \Sigma_{D}$ and $g_{j} \cap \Sigma_{D}=\varnothing$. We first show that if two $f_{i}$ 's coincide then $Y$ bounds a polyhedron of type 2 or 4.1 . We refer to Figure 21 for the notation.

Corollary 4.4 implies that two adjacent $f_{i}$ 's cannot coincide. Up to symmetry, the only cases we are left to deal with are $A$ - $\left(f_{1}=f_{3}\right), B$ - $\left(f_{1}=f_{3}\right)$, and $B$ $\left(f_{2}=f_{3}\right)$. In all cases we will show that $Y$ bounds a polyhedron of type 2 or 4.1 . The key point will be to exhibit two loops that must be fake because of Theorem 3.8.

Case $A$ - $\left(f_{1}=f_{3}\right)$ is seen in Figure 22-left: since $\alpha^{\prime}$ and $\alpha^{\prime \prime}$ are fake, one sees easily that $Y=\partial \mathcal{R}(\gamma)$, where $\mathcal{R}(\gamma)$ is a Möbius strip with two tongues (Figure 22-right).

Case $B$ - $\left(f_{1}=f_{3}\right)$ is similar; see Figure 23-left. We have $Y=\partial \mathcal{R}(\gamma)$, where $\mathcal{R}(\gamma)$ is an annulus with two tongues on opposite sides (Figure 23-right).

In case $B$ - $\left(f_{2}=f_{3}\right)$ we consider the loops of Figure 24-left. Since $\alpha^{\prime}$ and $\alpha^{\prime \prime}$ are fake we deduce that all the edges $e_{i} \cap \Sigma_{D}$ end at the same vertex $v$, such

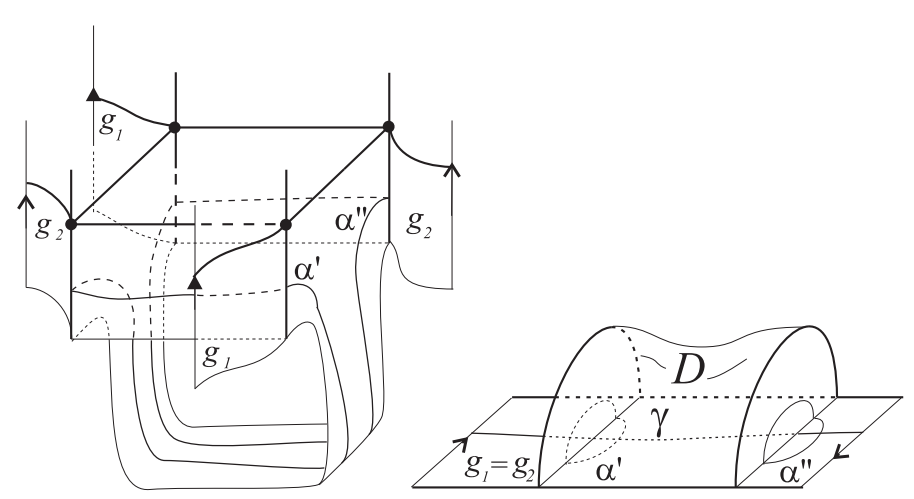

FIGURE 22. Proof of Fact 2: first case. 

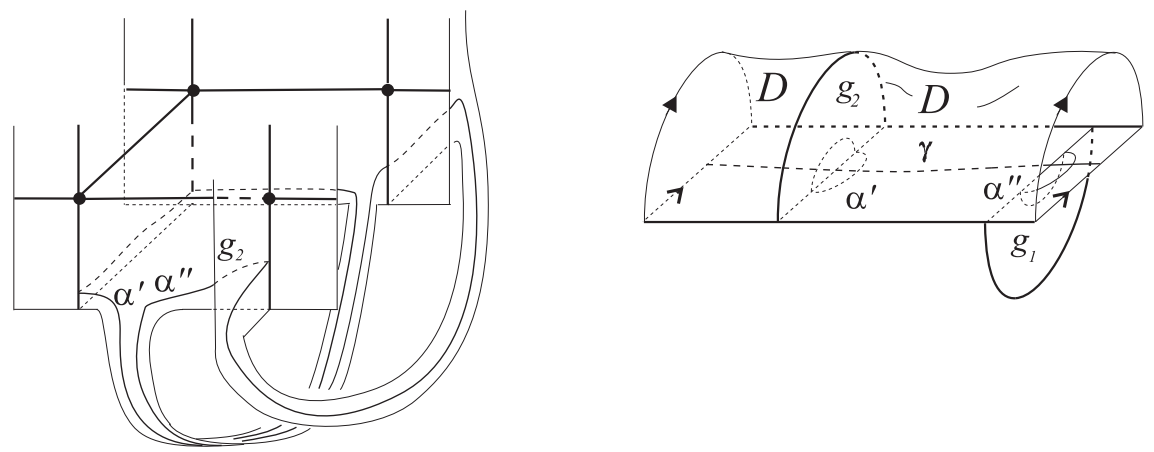

FIGURE 23. Proof of Fact 2: second case.
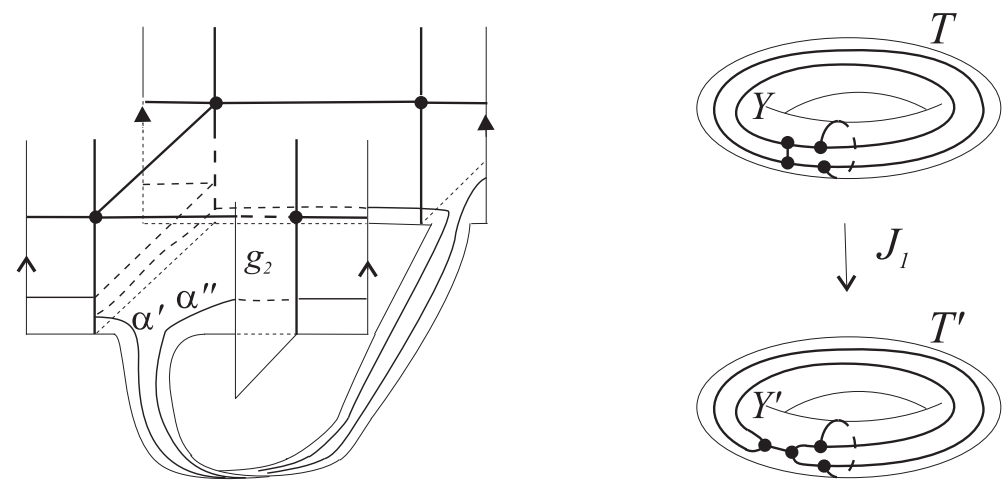

FIGURE 24. Proof of Fact 2: third case.

that $s_{2}, s_{3} \subset \operatorname{lk}(v)$. We can then apply a move $J_{1}$ whose effect on $Y$ is shown in Figure 24-right.

The result is a trace $Y^{\prime}$ which falls into case $A$ $\left(f_{1}=f_{3}\right)$. So $Y^{\prime}=\partial \mathcal{R}(\gamma)$ with $\mathcal{R}(\gamma)$ a Möbius strip with two tongues. Recalling that the inverse of a $J_{1^{-}}$ move is again a $J_{1}$-move, we only need to consider which such moves can be applied to $\mathcal{R}(\gamma)$. The move is determined by the edge of $\partial \mathcal{R}(\gamma)$ which disappears during the move: of the 6 edges of $\partial \mathcal{R}(\gamma), 4$ lead to a situation in which $e(D)=3$, so we exclude them. The other 2 edges are actually symmetric, and the result is of type 4.1 .

To conclude the proof of Fact 2 we must show that if the $f_{i}$ 's are distinct then $g_{1} \neq g_{2}$. If $Y$ is of type $B$ then $g_{1}$ has a certain component of $M \backslash(P \cup D)$ on both sides, and $g_{2}$ has the other one, so $g_{1} \neq g_{2}$. Assume in case $A$ that $g_{1}=g_{2}$. Referring to Figure 21 let $q_{j}$ be the midpoint of $t_{j}$, and join $q_{1}$ to $q_{2}$ by an arc $\lambda$ in $g_{1}=g_{2}$. There are 4 distinct arcs $\lambda_{1}, \ldots, \lambda_{4} \subset Y$ having endpoints $q_{1}$ and $q_{2}$ and intersecting $S(P)$ twice. For two of them the polyhedron $\mathcal{R}\left(\lambda \cup \lambda_{i}\right)$ is an annulus with 2 tongues on the same side. Then some $\lambda \cup \lambda_{i}$ is fake, which is in contrast with the fact that the $f_{i}$ 's are distinct.
Proof of Fact 3. We start with case $A$. Assume that $v_{1} \neq v_{2}$, and put $P^{\prime}=(P \cup D) \backslash f_{1}$. If $f_{1}$ is incident to $x$ different vertices of $P$ then $\# V\left(P^{\prime}\right)=$ $\# V(P)+4-2-x$. Since $P$ is minimal we have $x \leq 2$. On the other hand $f_{1}$ is incident to $v_{1}$ and $v_{2}$, so $x=2$. Now Figure 25 shows a triod $\tau$ in $P^{\prime}$, trace of a torus parallel to $F$. By Proposition 4.3, either $F$ is nonseparating or $\tau$ is boundary-parallel. In the first case we get a contradiction to the initial assumption. In the second case we deduce that $f_{1}$ is incident to $v_{3}$ and $v_{4}$, so $\left\{v_{3}, v_{4}\right\} \subset\left\{v_{1}, v_{2}\right\}$. So either $v_{3}=v_{4}$, or $v_{4}=v_{1}$, or $v_{3}=v_{1} \neq v_{4}=v_{2}$. In all cases but the last one the conclusion is the desired one up to symmetry. Concentrating on the

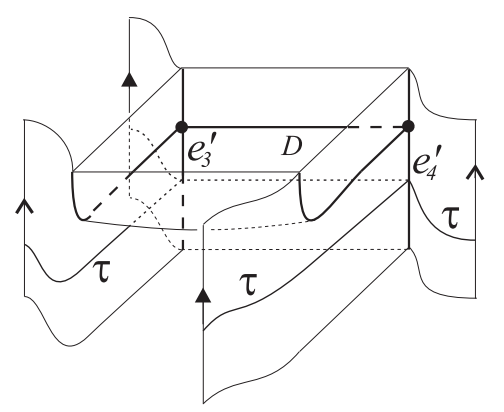

FIGURE 25. A triod $\tau \subset P^{\prime}$. 
last case, we note that $f_{1} \cup \cdots \cup f_{4}$ is a surface near $v_{1}$ and $v_{2}$, and that the $f_{i}$ 's and $g_{j}$ 's are all distinct. From these facts it is not hard to deduce that $v_{1}$ and $v_{2}$ appear as in Figure 26.

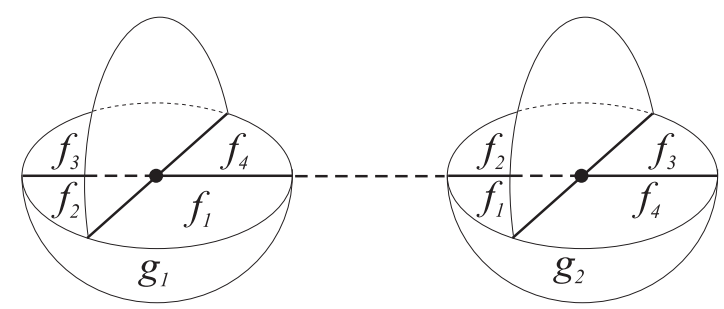

FIGURE 26. The vertices $v_{1}$ and $v_{2}$.

The figure readily implies that $f_{2}=f_{4}$ : a contradiction.

The proof in case $B$ is similar, except that $D$ cannot be used directly: a perturbed version $D^{\prime}$ as in Figure 27-left must be employed. We are again supposing here that $v_{1} \neq v_{2}$, so $f_{1}$ is incident to $x \geq 2$ vertices of $P$, but now $\# V\left(P^{\prime}\right)=\# V(P)+6-3-x$. Since $P$ is minimal we have $x \geq 3$, so $x \in\{2,3\}$. We first claim that we can suppose $x=3$ up to symmetry. By contradiction, assume that both $f_{1}$ and $f_{2}$ are incident to exactly 2 vertices. We deduce that the situation is as in Figure 27-right, where we also show a face $f$ incident twice to an edge, which is absurd by Corollary 4.4.

Our claim that $x=3$ up to symmetry is proved, so $\# V\left(P^{\prime}\right)=\# V(P)$ and $P^{\prime}$ is minimal too. A figure very similar to Figure 25 shows that a triod must exist in $P^{\prime}$, and allows to conclude as above that either $F$ is separating or $f_{1}$ is incident to $v_{3}$ and $v_{4}$. So either $v_{3}=v_{4}$, which gives the desired conclusion

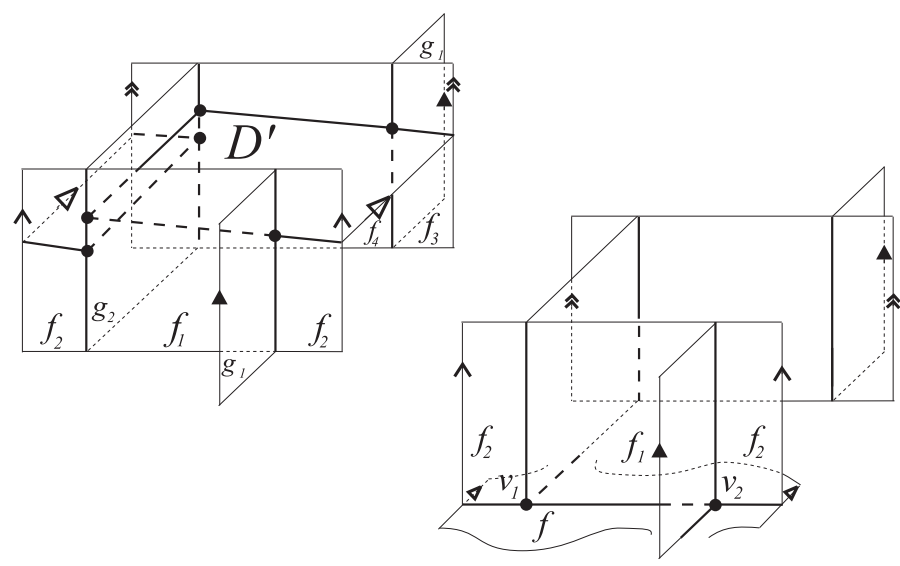

FIGURE 27. The perturbed disc $D^{\prime}$ (left), and a face $f$ doubly incident to an edge (right). up to symmetry, or $\left\{v_{3}, v_{4}\right\} \cap\left\{v_{1}, v_{2}\right\} \neq \varnothing$ (recall that $f_{1}$ is incident to exactly 3 vertices). If $v_{3}=v_{1}$ or $v_{4}=v_{2}$ we get the desired conclusion. Otherwise we can assume up to symmetry that $v_{1}=v_{4}$. So $e_{1}$ and $e_{4}$ have a common vertex in $P$, which implies that there is a face incident to both. But $e_{1}$ is adjacent to $f_{1}, f_{2}, g_{1}$ and $e_{4}$ is adjacent to $f_{3}, f_{4}, g_{2}$, and the $f_{i}$ 's and $g_{j}$ 's are distinct, so we get a contradiction.

Proof of Fact 4. If $Y$ is of type $A$, then $e\left(D^{\prime}\right)=3$, so by Fact 1 (and its proof) $Y^{\prime}$ bounds a polyhedron $Q$ of type 3.3 or of type 1 , but the latter is impossible because $Y^{\prime}$ is the trace of a torus. We only need to consider which $J_{1}$-moves can be applied to a $Q$ of type 3.3. By Lemma 4.12 the move actually takes place towards the exterior of $Q$ (that is, its result contains 2 vertices of $P$ ). The move is determined by the edge of $\partial Q$ which disappears during the move: of the 6 edges in $\partial Q, 3$ lead to a situation in which $e(D)=2$, so we exclude them. The other 3 edges are actually symmetric, and the result is a polyhedron of type 3.4 .

If $Y$ is of type $B$, then $u$ must be an edge in $\partial_{2} D$ (otherwise $e\left(D^{\prime}\right)=e(D)$ ), so $Y^{\prime}$ is of type $A$. Moreover $Y^{\prime}$ is the trace of a torus. Combining Fact 2 and the part of Fact 4 already established we see that $Y^{\prime}=\partial Q$ with $Q$ either of type 3.4 or a Möbius strip with two tongues (type 2). However, if we denote by $f_{i}^{\prime}$ the faces of $\Sigma_{D^{\prime}}$ incident to $D^{\prime}$, by Lemma 4.12 we have $f_{i}^{\prime} \subset f_{i}$ up to permutation, so the $f_{i}^{\prime \prime}$ 's are distinct. This shows that type 2 is impossible, and again we are left to analyze what can we get from a $Q$ of type 3.4 by a move $J_{1}$ which takes place towards the exterior. Of the 6 edges of $\partial Q, 4$ lead to a situation in which $e(D)=3$, so we exclude them. The other 2 edges are actually symmetric, and the result is type 3.5.

Proof of Fact 5. The first step of our proof is the extension of the move $Y \rightarrow Y^{\prime}$ to a flow $Y \rightarrow Y^{\prime} \rightarrow$ $Y^{\prime \prime} \rightarrow \cdots \rightarrow Y^{(k)}$ of $J_{1}$-moves. As mentioned in the proof of Fact 4 we must have $u \subset \partial_{1} D$ in this case, so we assume up to symmetry that $u=s_{1} \subset \partial f_{1}$, and we note that Remarks 4.10-4.11 and Lemma 4.12 apply. The situation is described in Figure 28.

One easily sees that the faces of $\Sigma_{D^{\prime}}$ incident to $\partial_{1} D^{\prime}$ are $f_{3}, f_{4}$ and two new ones (one of which is contained in $f_{2}$ ), which we denote by $f_{1}^{\prime}, f_{2}^{\prime}$. If $f_{1}^{\prime}$, $f_{2}^{\prime}, f_{3}, f_{4}$ are not distinct, the flow is reduced to 

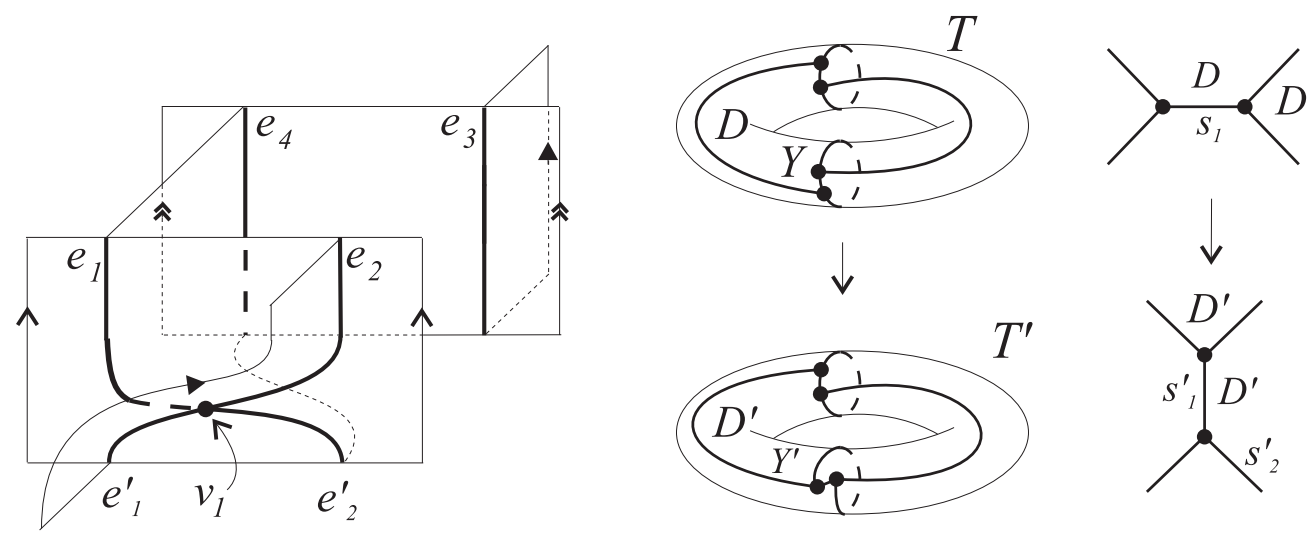

FIGURE 28. Proof of Fact 5.

$Y \rightarrow Y^{\prime}$, and we move to the next step. Otherwise let $v_{1}^{\prime}, v_{2}^{\prime}$ be the ends of $e_{1}^{\prime}, e_{2}^{\prime}$ (see Figure 28-left). If $v_{1}^{\prime} \neq v_{2}^{\prime}$ then again the flow is reduced to $Y \rightarrow Y^{\prime}$. Assume on the contrary that $v_{1}^{\prime}=v_{2}^{\prime}$, and consider Figure 28-right. Then either $s_{1}^{\prime}$ or $s_{2}^{\prime}$ is contained in $\operatorname{lk}\left(v^{\prime}\right)$, but certainly $s_{1}^{\prime}$ is not, for otherwise $P$ would contain an embedded face with two vertices, which is absurd by Corollary 4.4. Setting $u^{\prime}=s_{2}^{\prime}$, we are now in a position to apply a move $J_{1}$ along the triangle determined by $v_{1}^{\prime}$ and $u^{\prime}$, getting from $Y^{\prime}$ to $Y^{\prime \prime}$. We proceed in a similar way and note that the process must come to an end because $\Sigma_{D^{(i)}}$ contains one vertex less than $\Sigma_{D^{(i-1)}}$ by Lemma 4.12.

Our second step is to understand the final stage $Y^{(k)}$ of our flow. By construction either $f_{1}^{(k)}, f_{2}^{(k)}$, $f_{3}, f_{4}$ are not distinct or $v_{1}^{(k)} \neq v_{2}^{(k)}$. In the first case, since at each step only 1 face not contained in the previous one is inserted (and 1 is deleted), precisely 3 of $f_{1}^{(k)}, f_{2}^{(k)}, f_{3}, f_{4}$ are distinct. We know by Fact 2 (and its proof) that $Y^{(k)}$ (which is of type $B$ ) bounds a polyhedron $Q$ which is either an annulus with 2 tongues on opposite sides, or of type 4.1. The first case is excluded by what just said about the $f_{i}$ 's. By Lemma 4.12, $Y$ bounds $Q \cup_{Y^{(k)}}\left[Y, Y^{(k)}\right]$. Since at each step of the construction of our flow the choice of move $J_{1}$ was forced, the polyhedron $\left[Y, Y^{(k)}\right]$ is defined unambiguously (it depends on $k$ only). We only need to explain which edge of $\partial Q$ determines the $J_{1}$-move which glues $Q$ to $\left[Y, Y^{(k)}\right]$. Of the 6 edges, 2 lead to a trace of type $A, 2$ give rise to an embedded face with 2 edges (excluded by Corollary 4.4) and the other 2 are symmetric, so $Q \cup_{Y^{(k)}}\left[Y, Y^{(k)}\right]$ also depends on $k$ only. It is now a routine matter to check that indeed $Q \cup_{Y^{(k)}}\left[Y, Y^{(k)}\right]$ is the polyhedron of type 4.2 with $k$ vertices.
Having understood the case where $f_{1}^{(k)}, f_{2}^{(k)}, f_{3}, f_{4}$ are not distinct, we assume that they are. The rest of the proof is devoted to showing that it is actually impossible that $v_{1}^{(k)} \neq v_{2}^{(k)}$. Let us first assume that $v_{3} \neq v_{4}$. By Fact 3 we then have $v_{1}=v_{3}$ up to symmetry, and we can apply a move $J_{1}$ which reduces $e(D)$. Fact 4 shows that $Y^{(k)}$ bounds a polyhedron $Q$ of type 3.4 or 3.5 , but $\partial Q$ is of type $B$, so it must be of type 3.5. Once again we must analyze the possible results of a move $J_{1}$, towards the exterior of a $Q$ of type 3.5. Of the 6 edges of $\partial Q, 2$ lead to a trace of type $A$, and therefore are excluded. The 4 other edges come in 2 symmetric pairs. For one type, the result of the move $J_{1}$ contains an embedded face with 3 vertices, which is absurd by Corollary 4.4.

For the other type, the result contains an embedded face with 4 vertices. We can then apply a discreplacement move as in Figure 10, getting a new minimal skeleton $P^{\prime}$ of $(M, X)$. The evolution of the singular set is shown in Figure 29, where the two white dots lie on some $\theta_{i}$, the black dots are vertices, and the gray dots lie on $Y$. Since the edges leaving $\theta_{i}$ end at the same vertex, a $J_{1}$-move transforms $\theta_{i}$ into a triod which is not boundary-parallel. This contradicts Proposition 4.3.

We are left to deal with the case where $f_{1}^{(k)}, f_{2}^{(k)}$, $f_{3}, f_{4}$ are distinct, $v_{1}^{(k)} \neq v_{2}^{(k)}$, and $v_{3}=v_{4}$. In this case we can perform a $J_{1}$-move along either $s_{3}$ or $s_{4}$, and we can proceed just as above, constructing

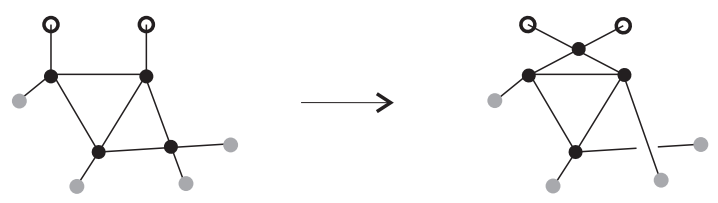

FIGURE 29. Proof of Fact 5, continued. 
a flow $Y^{(k)} \rightarrow Y^{(k+1)} \rightarrow \cdots \rightarrow Y^{(k+h)}$. During this process the faces $f_{1}^{(k)}, f_{2}^{(k)}$, and the vertices $v_{1}^{(k)}, v_{2}^{(k)}$ remain unaffected, while $f_{3}, f_{4}, v_{3}=v_{4}$ get transformed into $f_{3}^{(h)}, f_{4}^{(h)}, v_{3}^{(h)}, v_{4}^{(h)}$. As above, we have at the end of the sequence either that $f_{1}^{(k)}, f_{2}^{(k)}, f_{3}^{(h)}$, $f_{4}^{(h)}$ are not distinct or that $v_{3}^{(h)} \neq v_{4}^{(h)}$. In the first case, Fact 2 implies that $Y^{(k+h)}$ bounds a polyhedron of type 2 or 4.1. Such a polyhedron has at most 1 vertex, but $\Sigma_{D^{(k+h)}}$ contains at least $v_{1}^{(k)} \neq v_{2}^{(k)}$, and we get a contradiction. In the second case we are precisely in the situation $v_{3} \neq v_{4}$ previously considered, and again we get a contradiction.

This concludes the proof of Theorem 4.14.

\section{E. Conclusion of the Proofs}

If $Y$ is a trace in $P$, we denote by $P_{Y}$ the polyhedron $P \backslash \mathcal{R}(Y)$.

Proof of Theorem 3.3. Let $P$ be a minimal skeleton of $(M, X)$. By Corollary 2.8 we have $c(M, X)>0$, so $P$ is standard. Suppose a face $f$ of $P$ is incident to $\partial P$ in at least two distinct edges $e \subset \theta_{i}$ and $e^{\prime} \subset \theta_{i^{\prime}}$. We note that $i \neq i^{\prime}$ by Lemma 2.14 , and choose an arc $\alpha$ in $f$ having one end on $e$ and one on $e^{\prime}$. Then $Y=\partial \mathcal{R}\left(\theta_{i} \cup \theta_{i^{\prime}} \cup \alpha\right)$ is a trace with 4 vertices of a surface $F$. Moreover $P_{Y}=P_{1} \sqcup P_{2}$ is disconnected, so $F$ separates $M$ and hence it is orientable. Let $P_{2}$ be the component containing $\alpha$.

The graph $Y$ is of type $\mathrm{B}$ (see Figure 20) and $P_{2}$ has 3 boundary components (namely, $\theta_{i}, \theta_{i^{\prime}}$, and $Y)$. Now either $P_{1}$ or $P_{2}$ is of one of the types listed by Theorem 4.14, but no such type has 3 boundary components, so $P_{1}$ must be of one such type. The only polyhedra among those listed in Theorem 4.14 having at least one vertex and boundary of type B are those of type 3.5 (Figure 18) and 4 (Figure 19). If $P_{1}$ is of type 3.5 then $P$ is the skeleton of $B_{4}$, and if $P_{1}$ is of type 4 with 1 vertex then $P$ is the skeleton of $B_{3}$. Otherwise $P_{1}$ is of type 4 with $k \geq 2$ vertices, and the two edges of $S(P)$ adjacent to $\theta_{i}$ have a common endpoint. It easily follows that via a $J_{1^{-}}$ move we can transform $\theta_{i}$ into a triod which is not boundary-parallel and is the trace of a separating torus. This contradicts Proposition 4.3.

Proof of Theorem 3.5. Set $\mathcal{L}=\left\{\gamma_{1}, \ldots, \gamma_{n}\right\}$, where $\gamma_{i}$ is the core of the Möbius strip with one tongue attached to $\theta_{i} \subset \partial P$. By Theorem 3.3, even if we modify each $\gamma_{i}$ within its isotopy class, the $\gamma_{i}$ 's stay disjoint. Moreover, each $\mathcal{R}\left(\gamma_{i}\right)$ is a Möbius strip with one tongue. Therefore it is enough to show that $\mathcal{L}$ is a set of representatives of length-1 loops in $Q$. If not, there is a length-1 loop $\gamma$ not isotopic to any $\gamma_{i}$.

If $\gamma$ is disjoint from all $\gamma_{i}$ 's, then $\gamma \subset P$, so a face of $P$ is doubly incident to some edge, and we get a contradiction to Corollary 4.4. If $\gamma$ meets some $\gamma_{i}$ then, by Theorem 3.3, it meets only one, and we can assume that $\gamma \cap \gamma_{i}$ is one point away from $S(Q)$. Set $R=\mathcal{R}_{Q}\left(\gamma \cup \gamma_{i}\right)$. We need now to distinguish two cases, depending on whether $\mathcal{R}_{Q}(\gamma)$ is a Möbius strip or an annulus with one tongue. In the first case there exists a curve $\alpha$ contained in $\partial R$, and therefore in $P$, such that $l(\alpha)=2$ and $\alpha$ bounds an external disc ( $\alpha$ is homologous to $\gamma+\gamma_{i}$ in $R$ ). By Theorem $3.8 \alpha$ is fake, and it easily follows that $\gamma$ is isotopic to $\gamma_{i}$.

Assume now that $\mathcal{R}_{Q}(\gamma)$ is an annulus with one tongue. Note that $\partial R \subset P$ is a trace with 4 vertices of a separating, and hence orientable, surface $F$. Moreover $\partial R$ is of type $A$, so, by Theorem 4.14 , $\partial R$ bounds in $P$ a polyhedron $S$ of type 1.1, 3.3, 3.4, or 2 based on a Möbius strip. But $R \cap P$ is not of such a type, so the rest of $P$ is, hence $\# V(P) \leq 1$. But $\mathcal{B}_{\leq 1}^{1}=\left\{B_{0}, \ldots, B_{3}\right\}$, and we are done.

Before proving Theorem 3.7 we establish a general fact.

Lemma 4.15. Let $Q$ be a filling of a minimal skeleton $P$ of a brick distinct from $B_{0}, B_{1}, B_{2}, B_{3}, B_{4}$. Let $\left\{e_{1}, \ldots, e_{2 m}\right\}$ be a set of edges which disconnects $S(Q)$ in two components. Then there is a trace $Y$ contained in $P$ which has $2 m$ vertices $p_{i} \in e_{i}$ for $i=1, \ldots, 2 m$, and $Y$ is the trace of an orientable separating surface.

Proof. Take points $p_{i} \in e_{i}$; we have $S(Q) \backslash\left\{p_{i}\right\}=$ $K_{1} \sqcup K_{2}$. Let $f$ be a face of $Q$ incident to some $e_{i}$. The gluing path of $\partial f$ to $S(Q)$ can be split into $\operatorname{arcs} s_{1}, \ldots s_{2 \nu}$, meeting at points $q_{1}, \ldots, q_{2 \nu}$, where $s_{2 j+1} \subset K_{1}$ and $s_{2 j} \subset K_{2}$ for all $j$, and each $q_{k}$ is glued to one $p_{\beta(k)}$. The map $\beta$ is not necessarily injective, since $f$ can be multiply incident to an edge $e_{i}$. We can give the points $q_{k}$ alternating (red and black) colors.

Since $P=Q \backslash \mathcal{R}(\mathcal{L}(Q))$ is superstandard, $f$ can intersect at most one loop $\gamma$ among those in $\mathcal{L}(Q)$. 
Now take $\nu$ pairwise disjoint segments $\lambda_{1}, \ldots, \lambda_{\nu}$, properly embedded in $f$, such that

$$
\bigcup_{j=1}^{\nu} \partial \lambda_{j}=\bigcup_{k=1}^{2 \nu} q_{k} .
$$

We can ask the $\lambda_{j}$ 's to be disjoint from $\gamma$, since the points on $\partial f$ are separated into two even subsets by $\gamma$. It is easy to see that the two endpoints of each $\lambda_{j}$ automatically have distinct colours. If we do this for each face $f$ incident to some $e_{i}$, the union of all the chosen segments is a trace $Y$ disjoint from $\mathcal{L}(Q)$ and hence contained in $P$.

We claim that $Y$ has a product regular neighbourhood in $P$ : take for $i=1, \ldots, 2 m$ a vector $v_{i}$ at $p_{i}$, tangent to $e_{i}$ and directed towards $K_{2}$. Each segment of $Y$ is a $\lambda_{j}$, properly embedded in a face $f$ such that $\partial \lambda_{j}$ consists of points with distinct colors. It follows that the vectors at the ends of $\lambda_{j}$ extend along $\lambda_{j}$ to a non-vanishing field tangent to $f$. The existence of such a field on $Y$ easily implies that $F$ is orientable and that $F$ cuts $M$ into two components.

Proof of Theorem 3.7. Suppose $S(Q)$ contains a pair $\left\{e_{0}, e_{1}\right\}$ of separating edges. By Lemma 4.15 there is a trace $Y$ of a separating (and hence orientable) surface $F$ with two vertices, intersecting both $e_{0}$ and $e_{1}$. Proposition 4.3 applies, and possibility (1) is ruled out because $F$ separates. Both other possibilities imply that the vertices of $Y$ lie on the same edge of $Q$, but $e_{0} \neq e_{1}$ by assumption.

Suppose $S(Q)$ contains a separating quadruple $\left\{e_{0}, e_{1}, e_{2}, e_{3}\right\}$ of edges. By Lemma 4.15 there is a trace $Y$ of a separating (and hence orientable) surface with 4 vertices intersecting them. If $Y$ is connected then Theorem 4.14 applies, and we are done because the singular sets of polyhedra of types 1-4 indeed are as shown in Figure 5. If $Y=Y_{0} \sqcup Y_{1}$, then $Y_{0}$ is a trace with two vertices to which Proposition 4.3 applies. Now possibility (1) is ruled out either because every torus in $M$ is separating or by Theorem 4.6, and as above the other two possibilities lead to a contradiction.

\section{BRICKS AND SKELETA UP TO COMPLEXITY 9}

We provide in this section a complete description of the bricks in $\mathcal{B}_{n}$ for $n \leq 9$ anticipated in Section 1B.
Recall that $\mathcal{B}_{n}$ was split as $\mathcal{B}_{n}^{0} \sqcup \mathcal{B}_{n}^{1}$, where $\mathcal{B}_{n}^{0}$ consists of the elements of $\mathcal{B}_{n}$ without boundary. We describe now $\mathcal{B}_{\leq 9}^{1}$, postponing $\mathcal{B}_{\leq 9}^{0}$ for a moment, because to discuss it we will first need to introduce a new move on skeleta.

Our computations show that the set $\mathcal{B}_{\leq 9}^{1}$ consists of 11 bricks $B_{0}, \ldots, B_{10}$. Moreover, for $i \leq 9$ there is a unique minimal skeleton of $B_{i}$, while for $i=10$ there are two. Minimal skeleta for $B_{0}, \ldots, B_{4}$ were shown in Figures 2 and 4 , and for $B_{5}, \ldots, B_{10}$ they are now shown in Figure 30.
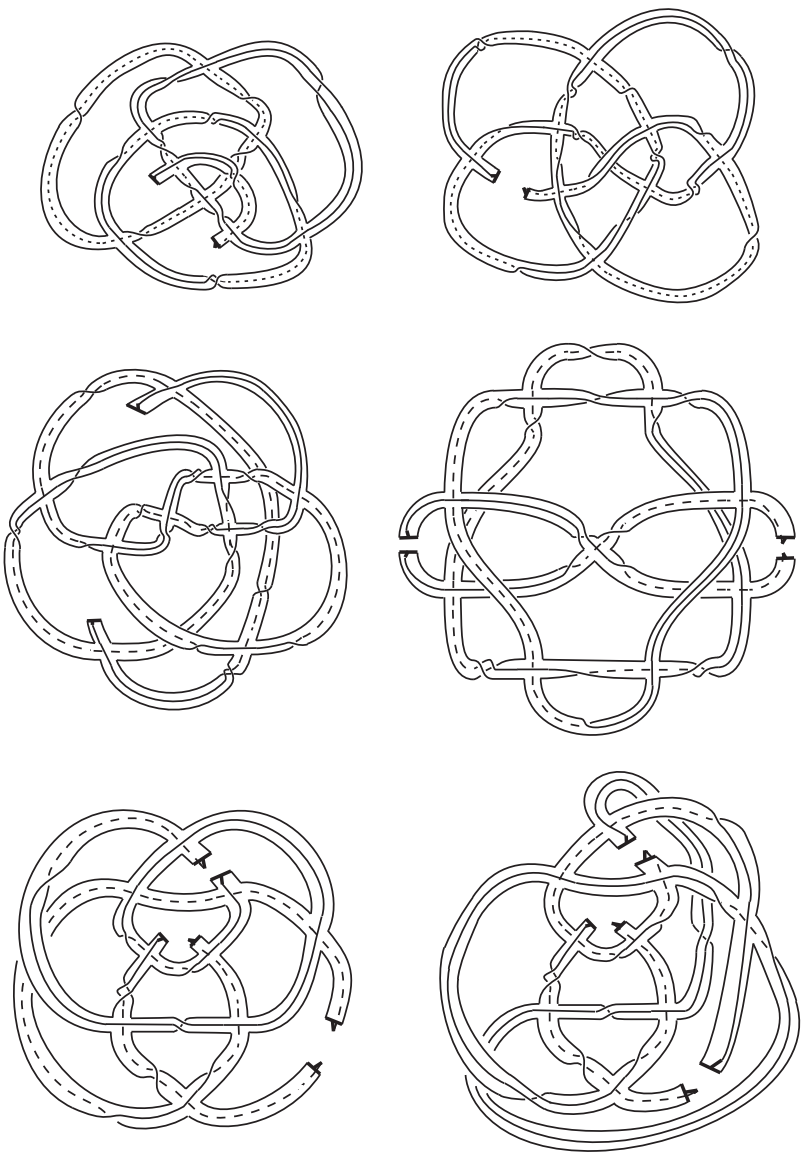

FIGURE 30. Minimal skeleta for $B_{5}, \ldots B_{10}$.

Using Remark 4.13, in this figure we only draw $\partial \mathcal{R}_{P}(S(P))$, and we use a thicker line for the $Y$ shaped portions of $\mathcal{R}_{P}(S(P))$ lying on $\partial P$. Each component of $\partial B_{i}$ contains two such $Y$ 's (shown close to each other when $\partial B_{i}$ has more than one component).

Having described $B_{0}, \ldots, B_{10}$, we can now prove Proposition 1.5. 
Proof of Proposition 1.5. Suppose $(M, \varnothing)$ is a sharp assembling of $B_{i}$ with $i \geq 6$ and some $B_{2}$ 's and $B_{3}$ 's. Since $c(M) \leq 9$, one $B_{3}$ can occur if $i=6$ only. Let $P_{i}$ be the minimal skeleton of $B_{i}$ shown in Figure 30. A minimal skeleton $P$ for $M$ is then a filling of $P_{i}$, possibly after glueing one copy of the minimal skeleton of $B_{3}$ if $i=6$. If we check all the polyhedra which can be built in this way, we see that many of them contain embedded faces with no more than 3 vertices, which contradicts Theorem 3.8. Only 16 of them do not contain such a face. Now 9 of these 16 are shown to be nonminimal by checking that small faces appear after suitable disc-replacement moves. The 7 polyhedra left out are skeleta of the 4 mentioned hyperbolic manifolds (there are some duplicates).

\section{A. Twists}

We introduce here a notion needed below to describe $\mathcal{B}_{\leq 9}^{0}$. Let $P$ be a quasi-standard skeleton of a closed manifold $(M, \varnothing)$, and let $\gamma$ be a length-2 loop in $P$ such that $\mathcal{R}(\gamma)$ is an annulus with 2 tongues. For $k \geq$ 1 let $W_{k}$ be the polyhedron of type 4 with $k$ vertices (Figure 19). The boundaries $\partial \mathcal{R}(\gamma)$ and $\partial W_{k}$ are homeomorphic (of type $B$ ). We can then choose a homeomorphism $\psi: \partial W_{k} \rightarrow \partial \mathcal{R}(\gamma)$ and form a polyhedron $P_{k}=(P \backslash \mathcal{R}(\gamma)) \cup_{\psi} W_{k}$. Note now that $W_{k}$ naturally sits in a solid torus $H$, with $\partial W_{k}=$ $W_{k} \cap \partial H$.

Proposition 5.1. The homeomorphism $\psi: \partial W_{k} \rightarrow$ $\partial \mathcal{R}(\gamma)$ can be chosen so that it extends to a homeomorphism $\Psi: \partial H \rightarrow \partial \mathcal{R}_{M}(\gamma)$. For these choices $P_{k}$ is a skeleton of the Dehn surgered manifold $M_{k}=$ $\left(M \backslash \mathcal{R}_{M}(\gamma)\right) \cup_{\Psi} H$
Proof. The first assertion is easy and we take it for granted. By construction $P_{k}$ sits in $M_{k}$ and it is simple, so we only need to show that $M_{k} \backslash P_{k}$ is an open 3-ball. To this end we note that $M \backslash(P \cup$ $\left.\mathcal{R}_{M}(\gamma)\right)$ is a ball $B$. Moreover $\partial H \backslash \partial W$ consists of two discs $D^{\prime}$ and $D^{\prime \prime}$, and $H \backslash(\partial H \cup W)$ consists of two balls $B^{\prime}$ and $B^{\prime \prime}$, with $\partial B^{\prime} \cap \partial H=D^{\prime}$ and $\partial B^{\prime \prime} \cap \partial H=D^{\prime \prime}$. So $M_{k} \backslash P_{k}=B \cup_{\left.\Psi\right|_{D^{\prime}}} B^{\prime} \cup_{\left.\Psi\right|_{D^{\prime \prime}}} B^{\prime \prime}$ is a ball.

We say that $P_{k}$ is obtained from $P$ by a $k$-twist along $\gamma$, and we adopt the convention that making a $0-$ twist means leaving $P$ unaffected.

\section{B. Closed Bricks up to Complexity 9}

Our computations show that the set $\mathcal{B}_{\leq 9}^{0}$ consists of 19 bricks which belong to the union of two classes $\left\{C_{i, j}\right\}$ and $\left\{E_{k}\right\}$. We describe here these manifolds and minimal skeleta $\tilde{C}_{i, j}$ and $\tilde{E}_{k}$ of them. (As opposed to the case of $\mathcal{B}_{n \leq 9}^{1}$, minimal skeleta are often not unique in $\mathcal{B}_{n \leq 9}^{0}$.) The polyhedron $\tilde{C}_{0,0}$ of Figure 31-left is a skeleton of $\left(S^{2} \times S^{1}, \varnothing\right)$ and it contains 2 length-2 loops $\gamma$ and $\delta$, shown in Figure 31left, such that $S^{2} \times S^{1} \backslash \mathcal{R}_{S^{2} \times S^{1}}(\gamma \cup \delta) \cong(A,(2,1))$, where $A$ is the annulus. Both $\mathcal{R}_{\tilde{C}_{0,0}}(\gamma)$ and $\mathcal{R}_{\tilde{C}_{0,0}}(\delta)$ are annuli with two tongues on different sides. We can therefore perform an $i$-twist along $\gamma$ and a $j$ twist along $\delta$. If we do this with appropriate gluing maps we get the skeleton shown in Figure 31-right, which we denote by $\tilde{C}_{i, j}$.

Using Proposition 5.1 it is not hard to check that $\tilde{C}_{i, j}$ is a skeleton of the Seifert manifold

$$
C_{i, j}=\left(S^{2},(2,1),(1+i, 1),(1+j, 1),(1,-1)\right) .
$$

We have $C_{i, j}=C_{j, i}$ for all $i, j$.

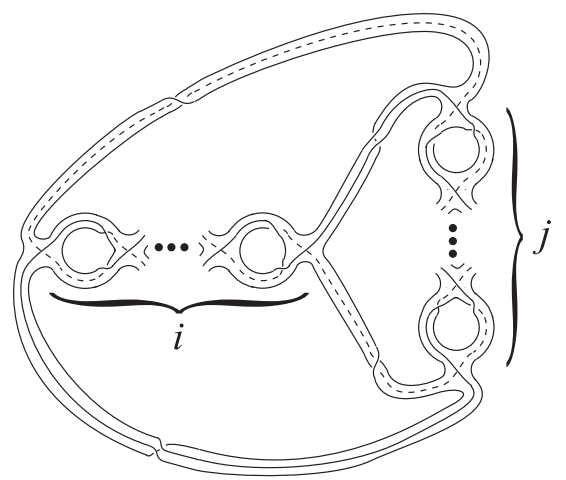

FIGURE 31. The length-2 loops $\gamma$ and $\delta$ in $\tilde{C}_{0,0}$ and the skeleton $\tilde{C}_{i, j}$. 

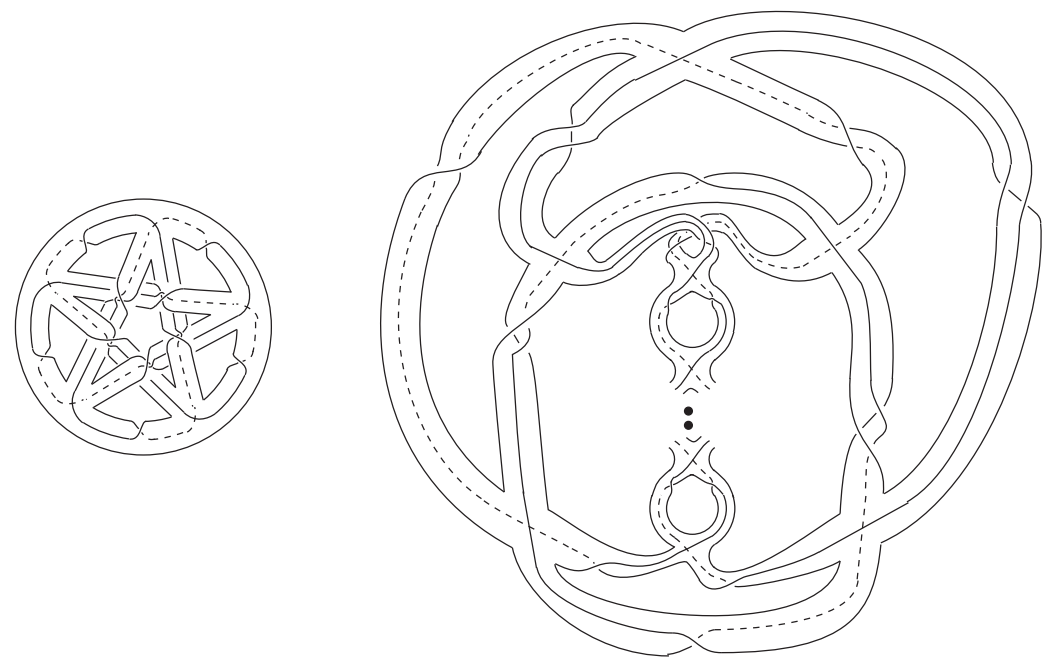

FIGURE 32. The skeleta $\tilde{E}_{0}$ and $\tilde{E}_{k}$.

Poincaré's homology sphere

$$
\left(S^{2},(2,1),(3,1),(5,1),(1,-1)\right)
$$

has a unique minimal skeleton $\tilde{E}_{0}$ (Figure 32-left). For any pair of non-adjacent edges of $S\left(\tilde{E}_{0}\right)$ there is a length-2 loop $\gamma$ intersecting them, isotopic to the singular fiber $(5,1)$. Since $\mathcal{R}(\gamma)$ is an annulus with two tongues, we can perform a $k$-twist along $\gamma$. If we do this with an appropriate gluing map we get the skeleton shown in Figure 32-right, which we denote by $\tilde{E}_{k}$. Each $\tilde{E}_{k}$ turns out to be a skeleton of the manifold $E_{k}=\left(S^{2},(2,1),(3,1),(5+k, 1),(1,-1)\right)$. It is worth mentioning here that the minimal skeleton of the brick $B_{5}$ may be obtained from $\tilde{E}_{0}$ by an operation similar to a $k$-twist along $\gamma$, except that the polyhedron of type 3.5 (Figure 18) is employed instead of $W_{k}$.

The set $\mathcal{B}_{\leq 9}^{0}$ consists of all manifolds $C_{i, j}$ and $E_{k}$ with $k \geq 0$ and $i \geq j \geq 1$ having at most 9 vertices (so $k \leq 4$ and $i+j \leq 9$ ), except the cases $k=1$ and $(i \geq 4, j=2)$. The skeleton $\tilde{E}_{1}$ is indeed minimal, but the associated manifold is not a brick, since it lies in $\left\langle B_{0}\right\rangle_{\text {self }}$. This is consistent with the fact that $\left(S^{2},(2,1),(3,1),(6,1),(1,-1)\right)$ fibers over $S^{1}$ with torus fiber. Each $\tilde{C}_{i, 0}$ is minimal (for $i \leq 9$ ), but the corresponding manifold is contained in $\left\langle B_{2}, B_{3}\right\rangle_{\text {nonself }}$. Each $\tilde{C}_{i, 2}$ for $i \geq 4$ is not minimal, since $\tilde{E}_{i-4}$ is a skeleton of the same manifold

$$
\left(S^{2},(2,1),(3,1),(i, 1),(1,-1)\right)
$$

with one fewer vertex.

\section{REFERENCES}

[Benedetti and Petronio 1995] R. Benedetti and C. Petronio, "A finite graphic calculus for 3-manifolds", Manuscripta Math. 88:3 (1995), 291-310.

[Callahan et al. 1999] P. J. Callahan, M. V. Hildebrand, and J. R. Weeks, "A census of cusped hyperbolic 3-manifolds", Math. Comp. 68:225 (1999), 321-332. With microfiche supplement.

[Hodgson and Weeks 1994] C. D. Hodgson and J. R. Weeks, "Symmetries, isometries and length spectra of closed hyperbolic three-manifolds", Experiment. Math. 3:4 (1994), 261-274.

[Matveev 1990] S. V. Matveev, "Complexity theory of three-dimensional manifolds", Acta Appl. Math. 19:2 (1990), 101-130.

[Matveev 1998] S. V. Matveev, "Tables of 3-manifolds up to complexity 6", preprint, Chelyabinsk University, 1998.

[Matveev and Fomenko 1988] S. V. Matveev and A. T. Fomenko, "Constant energy surfaces of Hamiltonian systems, enumeration of three-dimensional manifolds in increasing order of complexity, and computation of volumes of closed hyperbolic manifolds", Uspekhi Mat. Nauk 43:1(259) (1988), 5-22, 247. In Russian; translation in Russian Math. Surveys 43:1 (1988), 324.

[Ovchinnikov 1997] M. Ovchinnikov, "A table of closed orientable prime 3-manifolds of complexity 7", preprint, Chelyabinsk University, 1997.

[Turaev and Viro 1992] V. G. Turaev and O. Y. Viro, "State sum invariants of 3-manifolds and quantum $6 j$ symbols", Topology 31:4 (1992), 865-902. 
Bruno Martelli, Dipartimento di Matematica, Università di Firenze, Viale Morgagni 67a, 50134 Firenze, Italy (martelli@math.unifi.it)

Carlo Petronio, Dipartimento di Matematica Applicata, Università di Pisa, Via Bonanno Pisano 25/B 56126 Pisa, Italy (petronio@dm.unipi.it)

Received May 15, 2000; accepted in revised form November 2, 2000 